\title{
IDENTIFICATION OF TERMITE SPECIES BY THE HYDROCARBONS IN THEIR FECES
}

\author{
MICHAEL I. HAVERTY, ${ }^{1, *}$ R. JOSEPH WOODROW ${ }^{2}$ \\ LORI J. NELSON, ${ }^{1}$ and J. KENNETH GRACE ${ }^{2}$ \\ ${ }^{1}$ Pacific Southwest Research Station, Forest Service, U.S. Department of Agriculture, \\ Berkeley, CA 94701, USA \\ ${ }^{2}$ Department of Plant and Environmental Protection Sciences, University of Hawaii, \\ Honolulu, HI 96822, USA
}

(Received November 26, 2004; revised March 28, 2005; accepted May 1, 2005)

\begin{abstract}
Blends of abundant cuticular hydrocarbons are species-specific for termites (Isoptera) and can be used to identify a given taxon without the diagnostic castes, soldiers or adults. We demonstrate that hydrocarbon extracts of termite fecal pellets from damaged wood can also be characterized and used to identify termites responsible for damage, even though termites are no longer present or easily recovered. In structures infested by drywood termites, it is common to find fecal pellets, but difficult to extract termites from the finished wood in service. Nine species belonging to two families (Kalotermitidae and Termopsidae) were examined to compare the hydrocarbon composition of termites and their fecal pellets. Diversity was extensive: at least one half of the amount of the hydrocarbons from Neotermes connexus, Incisitermes immigrans, Cryptotermes brevis, Cryptotermes cynocephalus, Procryptotermes corniceps, and Zootermopsis nevadensis nuttingi was olefins. Incisitermes minor and Pterotermes occidentis incorporated only small amounts of olefins in cuticular hydrocarbons; Marginitermes hubbardi had no detectable olefins. Hydrocarbons extracted from fecal pellets were qualitatively and quantitatively similar to cuticular extracts and can be used to determine the termite species responsible without the termites present.
\end{abstract}

Key Words - Chemotaxonomy, cuticular hydrocarbons, dampwood termites, drywood termites, fecal pellets, frass, Kalotermitidae, Isoptera, species identification, Termopsidae.

* To whom correspondence should be addressed. E-mail: mhaverty@fs.fed.us 


\section{INTRODUCTION}

The cryptic nature of drywood and dampwood termites often precludes their collection for purposes of identification. Because they make their homes within wood, it is often not possible to collect them without destroying the wooden structure. A commonly utilized sign for determining the presence of wood-inhabiting termites is the associated occurrence of fecal pellets, which are ejected from the galleries inside the wood. These pellets are diagnostic for drywood and dampwood termites and can be used to distinguish their damage from other wood-destroying insects (Ebeling, 1975; Moore, 1992).

Drywood and dampwood termites excrete feces in the form of hard, evenly shaped fecal pellets. While collecting drywood termites in a survey of the termites of the Hawaiian Islands (Haverty et al., 2000; unpublished data), we gathered fecal pellets while removing termites from wood. Rather than simply signaling the general presence of termites, we wondered whether these pellets could be used for more precise diagnosis of the species of termite inhabiting the wood. We hypothesized that termite fecal pellets contain the same mixture of hydrocarbons as the insects that produced them, and because cuticular hydrocarbons are species-specific in termites (Page et al., 2002), we further hypothesized that the species can be identified by these chemicals in their feces.

The cuticle covering the outside surface of the termite also lines the inside of the rectum and likely also has a covering of wax composed mostly of hydrocarbons. Fecal pellets are formed in the elongate, bulbous rectum that is used for the temporary storage of undigested food particles (Child, 1934). Pellets are elongate and have six flattened sides that coincide with the rectal papillae. The six surfaces are a result of pressure applied by the six plates within the rectal epithelium. The hindgut is lined with chitinous intima that is continuous through the anus with the chitinous exoskeleton; the epithelial layer of the rectum is thickened and contains a heavily chitinous intima (Child, 1934).

Fecal pellets must be dehydrated before expulsion; resorption of water represents an essential ecological adaptation for xeric conditions (Noirot and Noirot-Timothée, 1969). The function of the rectum is to press out and conserve the water content of material that enters from the colon. Water is absorbed through the rectal wall. During compression for removal of water, it is likely that pellets acquire a hydrocarbon coating before extrusion. This may facilitate discharge of pellets.

Characterization of the cuticular hydrocarbons of termite species supports the concept of species specificity of hydrocarbon mixtures (Haverty et al., 1988, 1996a, 1997, 1999, 2000; Watson et al., 1989; Kaib et al., 1991; Brown et al., 
1994, 1996; Haverty and Nelson, 1997; Takematsu and Yamaoka, 1999; Clément et al., 2001; Nelson et al., 2001; Page et al., 2002). Owing to the ontogenic origin of the insect hindgut (Snodgrass, 1935; Romoser and Stoffolano, 1988), i.e., invaginated ectoderm, we hypothesized that external (cuticular) hydrocarbons are also present internally and are picked up by expelled fecal material.

\section{METHODS AND MATERIALS}

Collection of Termites. Termites were gathered from the British Virgin Islands (Haverty et al., 1997) and the Hawaiian Islands (Haverty et al., 2000). Collections of Pterotermes occidentis (Walker) and Marginitermes hubbardi (Banks) were made in the vicinity of Tucson, AZ, USA, and collections of Zootermopsis nevadensis nuttingi Haverty and Thorne were made near Mt. Shasta, CA, USA. In total, nine species belonging to two families were collected. Representatives of the Kalotermitidae included Neotermes connexus Snyder, Incisitermes immigrans Snyder, Cryptotermes brevis (Walker), Cryptotermes cynocephalus Light, Incisitermes minor (Hagen), Procryptotermes corniceps (Snyder), P. occidentis, and M. hubbardi; Z. nevadensis nuttingi was the sole representative of the Termopsidae.

Naturally infested wood samples were bagged and brought to the laboratory, where termites and fecal pellets were separated from wood and other debris. Samples of 15-20 pseudergates or nymphs were placed in separate vials, frozen, and dried (Haverty et al., 1997, 2000). As soon as the termites were completely dry, specimens were placed in vials that were tightly capped. Fecal pellets were separated from debris by sequentially sifting them through successively smaller sieves. Pellets were separated from fine debris by removing them with forceps or with an aspirator until 3-10 ml were obtained. Concurrently, fresh (i.e., not dried) termite voucher samples were preserved in $85 \%$ ethanol and deposited in the National Museum of Natural History, Smithsonian Institution, Washington, DC (Haverty et al., 1997) or in the University of Hawaii, Manoa Campus collection (Haverty et al., 2000).

Extraction Procedure and Characterization of Hydrocarbons. Hydrocarbons from the termites and fecal pellets were extracted, characterized, and quantified as reported in Haverty et al. (1997). Termites, as a group, or 3-10 ml of fecal pellets were extracted by immersion in $10 \mathrm{ml}$ of $n$-hexane for $10 \mathrm{~min}$. After extraction, hydrocarbons were separated from other compounds by pipetting the extract through $4 \mathrm{~cm}$ of activated Sigma silica gel (70-230 mesh) in Pasteur pipet minicolumns. An additional $5 \mathrm{ml}$ of clean $n$-hexane were 
dripped through the silica gel. The resulting extracts were evaporated to dryness under a stream of nitrogen and redissolved in $60 \mu \mathrm{l}$ of $n$-hexane for gas chromatography-mass spectrometry (GC-MS) analyses. A 3- $\mu$ l aliquot was injected into the GS-MS.

GC-MS analyses were performed on a Hewlett-Packard (HP) 5890 gas chromatograph equipped with an HP 5970B Mass Selective Detector interfaced with HP Chemstation data analysis software (HP59974J Rev. 3.1.2). The GCMS was equipped with an HP-1 fused silica capillary column $(25 \mathrm{~m} \times 0.2 \mathrm{~mm}$ ID) and operated in split mode (with a split ratio of 8:1). Each mixture was analyzed by a temperature program ranging from 200 to $320^{\circ} \mathrm{C}$ at $3^{\circ} \mathrm{C} / \mathrm{min}$ with a final hold of 11 or 16 min. Electron impact (EI) mass spectra were obtained at $70 \mathrm{eV}$.

$n$-Alkanes were identified by their mass spectra. Mass spectra of methylbranched alkanes were interpreted as described by Blomquist et al. (1987) to identify methyl branch locations. Mass spectra of di- and trimethylalkanes were interpreted as described by Page et al. (1990a,b) and Pomonis et al. (1978). Alkenes were identified by their mass spectra, but double bond positions were not determined (see Haverty et al., 1997). Equivalent chain lengths (ECL) were calculated for some of the unsaturated compounds to distinguish the various isomers.

In the text and tables, we use shorthand nomenclature to identify individual hydrocarbons or mixtures of hydrocarbons. This shorthand uses a descriptor for the location of methyl groups (X-me), the total number of carbons (CXX) in the hydrocarbon component excluding the methyl branch(es), and the number of double bonds following a colon (CXX:Y). Thus, heptacosane becomes $n-\mathrm{C} 27$; 2-methylheptacosane becomes 2-meC27; 13,15-dimethylheptacosane becomes 13,15-dimeC27; and heptacosadiene becomes C27:2. Hydrocarbons are presented in the tables for each species and its feces in the order of elution on our GC/MS system.

Integration of the total ion chromatogram was performed using the HP Chemstation data analysis software. GC-MS peak areas were converted to percentages of the total hydrocarbon fraction. A summary of the relative amounts of each peak for termites and fecal pellets using a representative chromatogram of each species was prepared.

Cluster Analysis of Hydrocarbon Mixtures from Termites and Fecal Pellets. The percentage of each hydrocarbon was used as the response variable. The presence of coeluting compounds precluded exact quantification of many individual hydrocarbons; the quantity of each hydrocarbon in each such peak was equal to $1 / n$ of the value for the peak, with $n=$ the number of hydrocarbons in the peak. The Euclidean distance for all 18 combinations of termite species by insect or fecal pellets was calculated using all hydrocarbons (R Statistical Language, 2004). 


\section{RESULTS AND DISCUSSION}

The cuticular hydrocarbons for five species $[N$. connexus, C. brevis, $C$. cynocephalus, I. immigrans, and I. minor] from Hawaii (Haverty et al., 2000), and one additional species $[P$. corniceps] from the British Virgin Islands (Haverty et al., 1997) were previously characterized. The composition of the hydrocarbon mixtures for these six species and the hydrocarbons from their fecal pellets are displayed in Figures 1-6 and summarized in Tables 1-6.
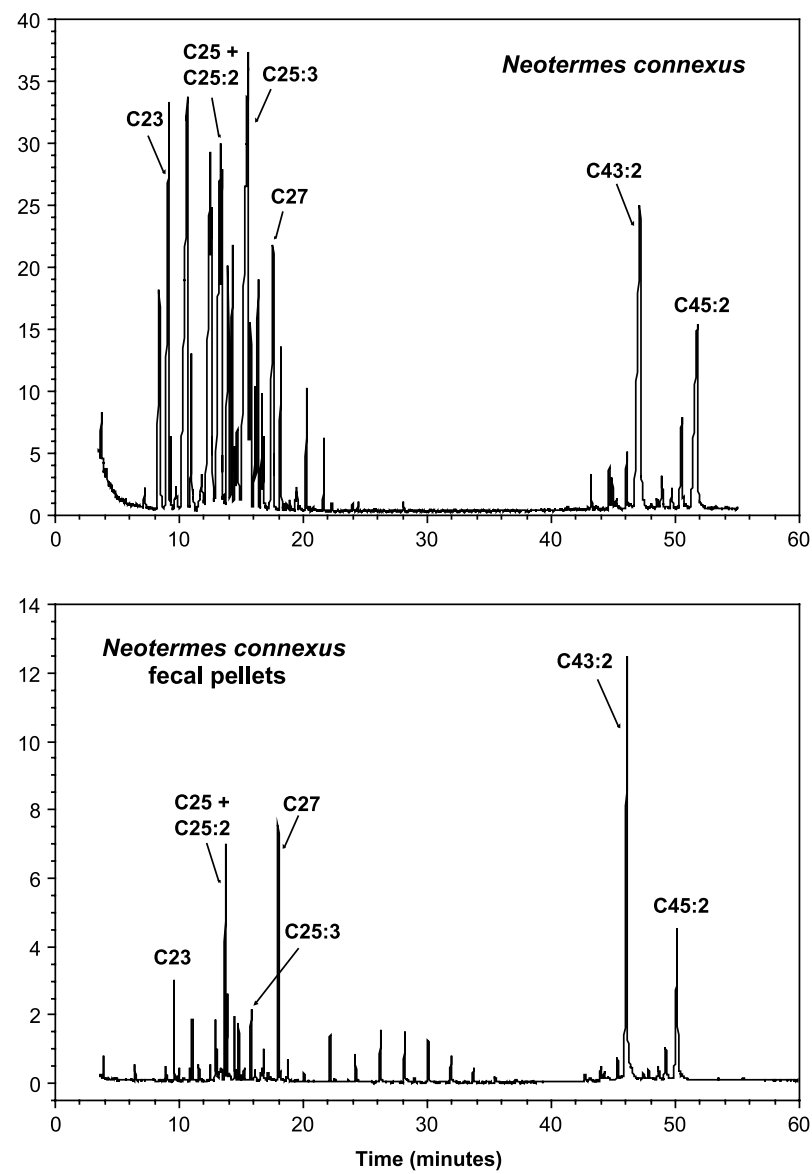

FIG. 1. Total ion chromatogram of the cuticular hydrocarbons from nymphs of Neotermes connexus Snyder from Iao Valley Lookout, Maui, HI, and from fecal pellets of $N$. connexus from junctions of Hwy 550 and 552, Kauai, HI. 

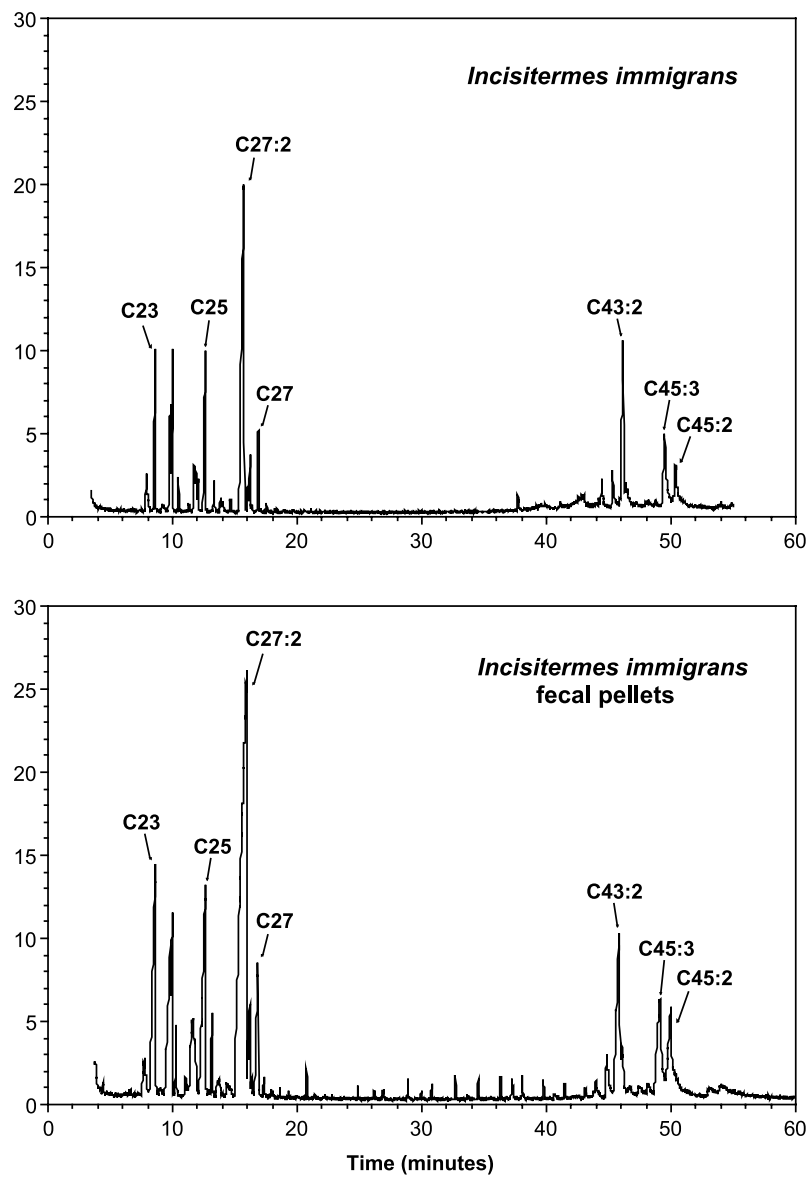

FIG. 2. Total ion chromatogram of cuticular hydrocarbons from pseudergates of Incisitermes immigrans Snyder from Poipu Beach, Kauai, HI, and from fecal pellets of I. immigrans from Kualoa Beach, Oahu, HI.

Detail for the cuticular hydrocarbon mixtures of two additional drywood termite species, P. occidentis and M. hubbardi, from Arizona, as well as the hydrocarbons from their fecal pellets, that have not been previously reported are shown (Figures 7 and 8, Tables 7 and 8). The composition of the cuticular hydrocarbons for frozen samples of $Z$. nevadensis nuttingi (Zootermopsis Phenoytpe III) has been described previously (Haverty et al., 1988). Here, we identify additional hydrocarbons not previously detected, as well as hydrocarbons from fecal pellets (Figure 9, Table 9). The results of the method used 

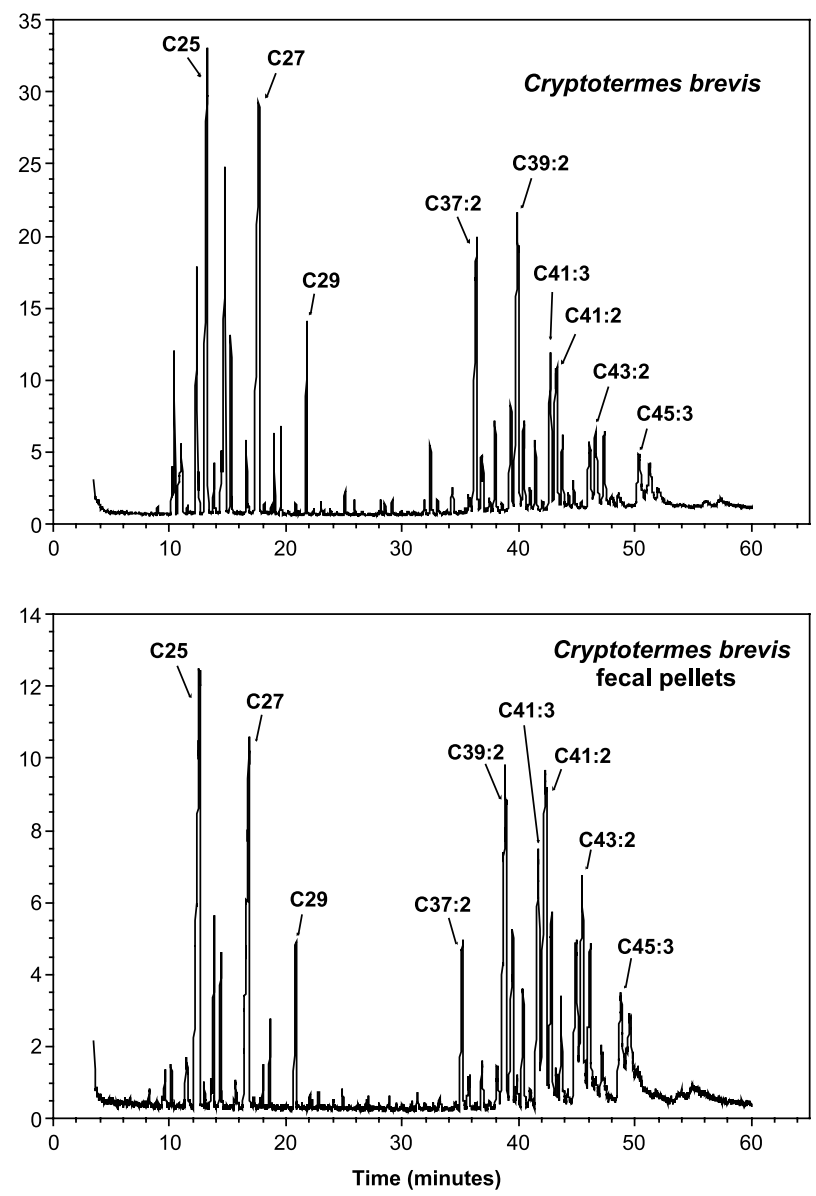

FIG. 3. Total ion chromatogram of the cuticular hydrocarbons from pseudergates of Cryptotermes brevis (Walker) from Honolulu, Oahu, HI, and from fecal pellets of C. brevis from NOAA laboratory, Kewalo Basin, Oahu, HI.

here are not equivalent to extracting live or freshly frozen termites, and demonstrate that there are quantitative differences in the composition of cuticular hydrocarbons extracted from dried specimens (Haverty et al., 1996b).

The cuticular hydrocarbon mixture of most drywood termite species examined thus far from the West Indies and Hawaii (Haverty et al., 1997, 2000) reflected a general pattern. Cuticular hydrocarbons occurred in two distinct groups: early eluting compounds (23 to 29 or 31 carbons in the parent chain) and late-eluting compounds ( 37 to 45 carbons in the parent chain). The mixtures 

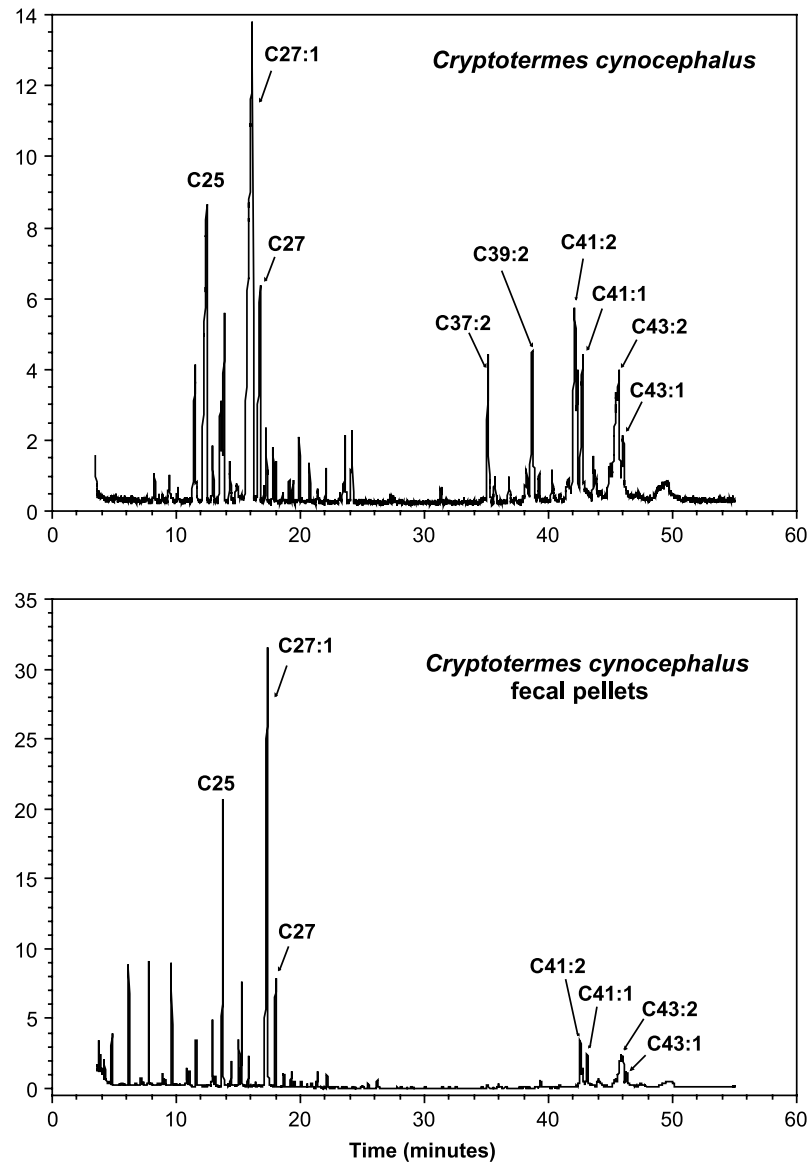

FIG. 4. Total ion chromatogram of the cuticular hydrocarbons from pseudergates of Cryptotermes cynocephalus Light from Waiahole Valley Road, Kamehameha Highway, Oahu, HI, and from fecal pellets from the same collection.

of I. minor and M. hubbardi were different in that hydrocarbons occurred continuously from the early eluting hydrocarbons with 23 carbons in the parent chain to the late eluting hydrocarbons with 43 carbons in the parent chain (Figures 5 and 8). Z. nevadensis nuttingi displayed a pattern similar to the general pattern for drywood termites with the addition of a few hydrocarbons with 31 carbons in the parent chain (Figure 9).

Hydrocarbons from Termites. $N$. connexus is generally found in rotting wood or living trees in moist, forested areas in Hawaii between 200 and $2000 \mathrm{~m}$ in elevation (Zimmerman, 1948). The chromatograms of cuticular hydrocarbons 

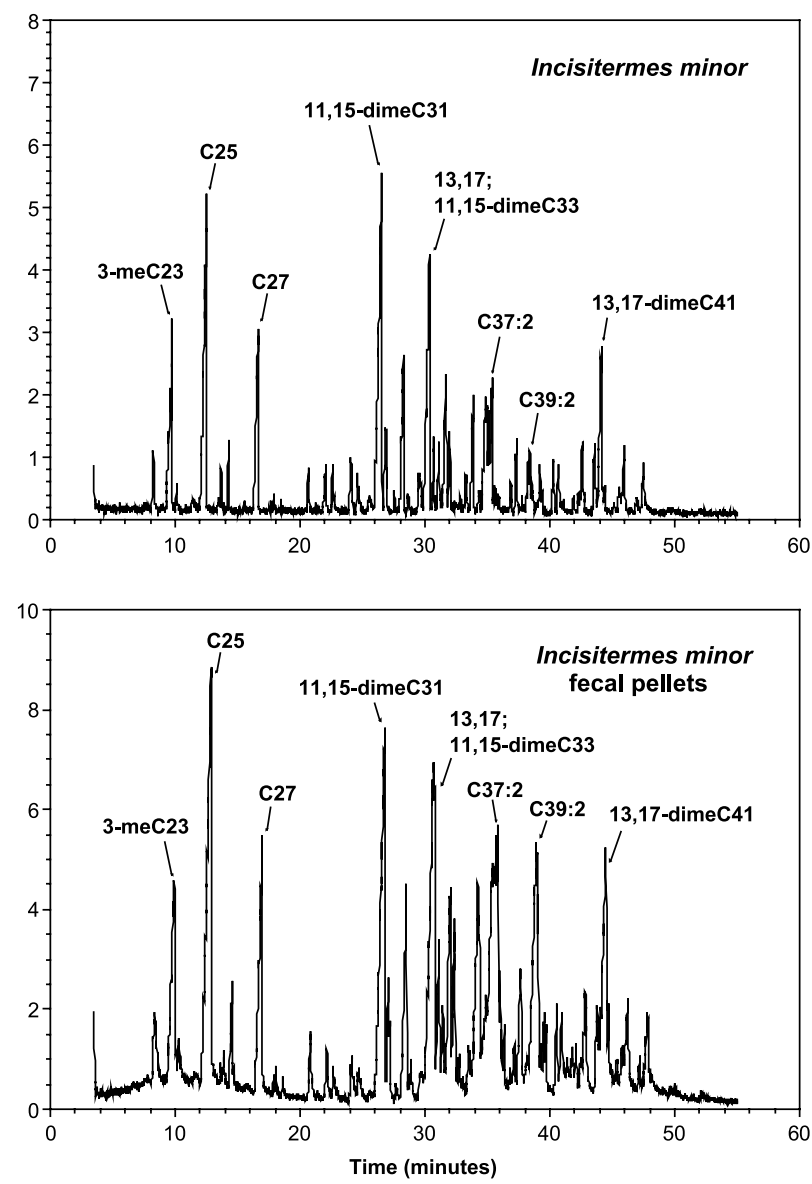

FIG. 5. Total ion chromatogram of cuticular hydrocarbons from pseudergates of Incisitermes minor (Hagen) from Fresno, CA, and from fecal pellets from the same collection.

reflected the general pattern of drywood termites (Figure 1, Table 1). n-Alkanes comprised $21.8 \%$ of the total hydrocarbon; $n$-C23, $n$-C25, and $n$-C27 were the most abundant. Olefins were, by far, the most predominant, representing over $52 \%$ of the total hydrocarbon; dienes and trienes were the most abundant olefins. Terminally branched monomethylalkanes, mainly 2-; 3-meC23; and 2meC24, accounted for $23.6 \%$ of the total hydrocarbon. Internally branched monomethylalkanes were rare; neither dimethylalkanes nor trimethylalkanes were detected. 

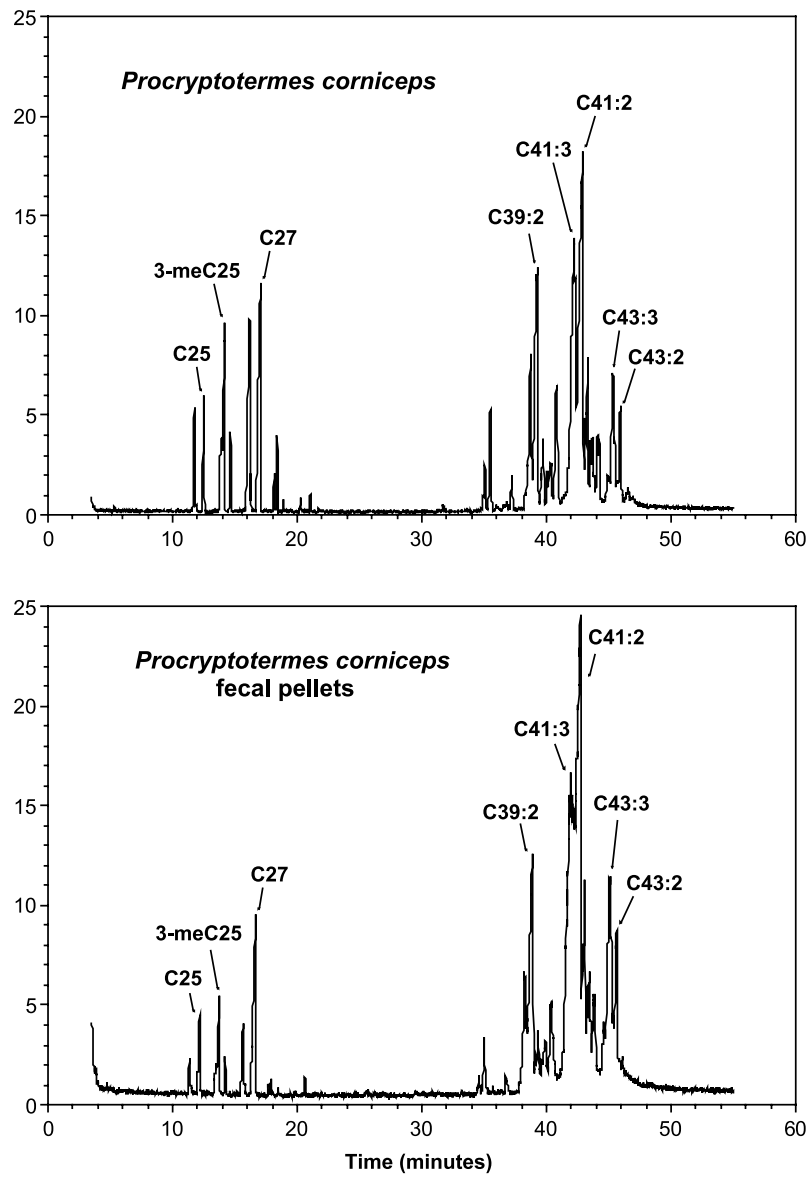

FIG. 6. Total ion chromatogram of the cuticular hydrocarbons from pseudergates of Procryptotermes corniceps (Snyder) from Guana Island, British Virgin Islands, and fecal pellets from the same collection.

I. immigrans is the predominant drywood termite in lowland xeric and coastal littoral forests, where it infests living and dead, standing trees, exclusive of man-made structures (Zimmerman, 1948). It is displaced by $N$. connexus at higher elevations, and the two species coexist at low elevations on mesic sites. The general appearance of the chromatograms of I. immigrans (Figure 2) was similar to those of $N$. connexus (Figure 1); over $75 \%$ of the hydrocarbons had 23 to 29 carbons (Table 2). $n$-Alkanes, primarily $n$-C25 and $n$-C27, comprised $24.8 \%$ of the total hydrocarbon. The unsaturated components constituted $59.4 \%$ 
TABle 1. Relative ABUndancE ${ }^{a}$ OF Hydrocarbons From NyMPHS AND FeCAL PELLETS OF Neotermes connexus SNYDER

\begin{tabular}{|c|c|c|c|}
\hline Hydrocarbons & $\mathrm{ECL}^{b}$ & Termites & Fecal pellets \\
\hline$n-\mathrm{C} 22$ & & 0.21 & 0.10 \\
\hline $2-\mathrm{meC} 22$ & & 3.36 & 0.38 \\
\hline $\mathrm{C} 23: 1$ & 22.70 & 0.00 & 0.19 \\
\hline$n-\mathrm{C} 23$ & & 7.26 & 2.59 \\
\hline $\mathrm{C} 23: 2$ & 23.01 & 0.37 & 0.00 \\
\hline Unknown & & 0.00 & 0.38 \\
\hline 9-meC23 & & 0.34 & 0.12 \\
\hline $2-; 3-\mathrm{meC} 23^{c}$ & & 9.75 & 2.11 \\
\hline$n$-C24 & & 1.23 & 0.47 \\
\hline $\mathrm{C} 24: 2$ & 24.01 & 0.20 & 0.00 \\
\hline 3,7-dimeC23 & & 0.00 & 0.17 \\
\hline $\mathrm{C} 25: 2$ & 24.50 & 0.69 & 0.54 \\
\hline 2-meC24 & & 8.00 & 2.07 \\
\hline $\mathrm{C} 25: 1$ & 24.70 & 1.67 & 1.03 \\
\hline $\mathrm{C} 25: 3+$ Unknown $^{c}$ & 24.80 & 0.41 & 0.00 \\
\hline $\mathrm{C} 25: 1$ & 24.80 & 0.00 & 1.29 \\
\hline$n-\mathrm{C} 25$ & & 8.40 & 7.26 \\
\hline $\mathrm{C} 25: 2$ & 25.01 & 2.17 & 2.42 \\
\hline Unknown & & 0.00 & 0.42 \\
\hline $\mathrm{C} 25: 3$ & 25.20 & 0.10 & 0.00 \\
\hline $\mathrm{C} 25: 2$ & 25.30 & 3.11 & 1.99 \\
\hline $\mathrm{C} 25: 2$ & 25.35 & 0.81 & 0.37 \\
\hline $\mathrm{C} 25: 2$ & 25.50 & 2.91 & 1.79 \\
\hline $2-\mathrm{meC} 25$ & & 0.63 & 0.20 \\
\hline 3-meC25 & & 1.25 & 0.54 \\
\hline $\mathrm{C} 25: 3$ & 25.98 & 15.38 & 2.95 \\
\hline $\mathrm{C} 25: 3$ & 26.07 & 1.29 & 0.58 \\
\hline $\mathrm{C} 25: 3$ & 26.10 & 0.89 & 0.00 \\
\hline $\mathrm{C} 26: 2 ; \mathrm{C} 27: 2 ; \mathrm{C} 25: 3^{c}$ & 26.30 & 1.07 & 0.54 \\
\hline $\mathrm{C} 25: 3$ & 26.47 & 2.56 & 1.17 \\
\hline 2-meC26; $\mathrm{C} 27: 1^{c}$ & 26.70 & 1.02 & 0.34 \\
\hline $\mathrm{C} 27: 1$ & 26.85 & 0.53 & 0.18 \\
\hline$n-\mathrm{C} 27$ & & 4.09 & 8.16 \\
\hline $\mathrm{C} 27: 2$ & 27.01 & 0.00 & 0.33 \\
\hline $\mathrm{C} 27: 2 ; 13-; 11-\mathrm{meC} 27^{c}$ & 27.35 & 1.79 & 0.89 \\
\hline $\mathrm{C} 27: 2$ & 27.50 & 0.05 & 0.00 \\
\hline $\mathrm{C} 28: 2$ & 27.60 & 0.09 & 0.00 \\
\hline 3-meC27 & & 0.08 & 0.00 \\
\hline $\mathrm{C} 27: 3$ & 27.85 & 0.15 & 0.00 \\
\hline$n-\mathrm{C} 28$ & & 0.12 & 0.31 \\
\hline $\mathrm{C} 29: 2$ & 28.50 & 0.94 & 0.00 \\
\hline$n-\mathrm{C} 29$ & & 0.47 & 1.61 \\
\hline$n-\mathrm{C} 30$ & & 0.00 & 0.99 \\
\hline$n-\mathrm{C} 31$ & & 0.00 & 1.85 \\
\hline$n-\mathrm{C} 32$ & & 0.00 & 1.82 \\
\hline
\end{tabular}


TABLE 1. CONTINUED

\begin{tabular}{|c|c|c|c|}
\hline Hydrocarbons & $\mathrm{ECL}^{b}$ & Termites & Fecal pellets \\
\hline$n-\mathrm{C} 33$ & & 0.00 & 1.66 \\
\hline$n-\mathrm{C} 34$ & & 0.00 & 1.10 \\
\hline$n-\mathrm{C} 35$ & & 0.00 & 0.67 \\
\hline$n-\mathrm{C} 36$ & & 0.00 & 0.32 \\
\hline$n-\mathrm{C} 37$ & & 0.00 & 0.11 \\
\hline $\mathrm{C} 41: 2$ & & 0.27 & 0.35 \\
\hline $\mathrm{C} 41: 1$ & & 0.00 & 0.28 \\
\hline 15-meC41 & & 0.38 & 0.79 \\
\hline $\mathrm{C} 42: 2$ & & 0.33 & 0.54 \\
\hline $\mathrm{C} 43: 3$ & & 0.61 & 1.20 \\
\hline $\mathrm{C} 43: 2$ & & 8.08 & 27.95 \\
\hline $15-\mathrm{meC} 43$ & & 0.12 & 0.39 \\
\hline $\mathrm{C} 44: 2$ & & 0.42 & 0.64 \\
\hline $\mathrm{C} 45: 4$ & & 0.25 & 0.86 \\
\hline $\mathrm{C} 45: 3$ & & 1.46 & 2.43 \\
\hline $\mathrm{C} 45: 2$ & & 4.70 & 12.55 \\
\hline
\end{tabular}

${ }^{a}$ Percent of total hydrocarbon composition.

${ }^{b}$ Equivalent chain length.

${ }^{c}$ An isomeric mixture or two or more compounds coelute in this peak.

of the total hydrocarbon; olefins with 27 or 43 carbons predominate. The terminally branched monomethylalkanes comprised $14.4 \%$ of the total hydrocarbon. Small amounts of internally branched monomethylalkanes were detected; di- and trimethylalkanes were absent.

C. brevis is the common drywood termite found in structures in Hawaii; its distribution is pantropical. It is almost exclusively found in buildings and furnishings. The hydrocarbon mixture of $C$. brevis resembles the general pattern of drywood termites living in xeric, tropical habitats (Haverty et al., 1997). Hydrocarbons occurred in two groups: the early eluting compounds consisted almost exclusively of $n$-alkanes and terminally branched monomethyl alkanes, whereas late-eluting compounds were chiefly olefins (Figure 3, Table 3). $n$ Alkanes, primarily $n$-C25 and $n$-C27, comprised ca. $29.4 \%$ of the total hydrocarbon. Alkenes, alkadienes, and alkatrienes were the predominant class, representing over $50 \%$ of the total hydrocarbon. The terminally branched monomethylalkanes amounted to $16 \%$ of the total hydrocarbon. Internally branched monomethylalkanes and dimethylalkanes occurred in small quantities.

C. cynocephalus was recently discovered in native vegetation in Hawaii (Haverty et al., 2000; Scheffrahn et al., 2000; Grace et al., 2002), and is found in structures in the Philippine Islands (Scheffrahn et al., 2000). This species also reflects the general pattern of hydrocarbon mixtures of drywood termites living in xeric, tropical habitats (Haverty et al., 1997) and is similar to C. brevis. 
TABle 2. Relative ABUndancE ${ }^{a}$ OF Hydrocarbons From PseUdergates AND FECAL PELlETS OF Incisitermes immigrans SNYDER

\begin{tabular}{|c|c|c|c|}
\hline Hydrocarbons & $\mathrm{ECL}^{b}$ & Termites & Fecal pellets \\
\hline 2-meC22 & & 1.65 & 1.37 \\
\hline$n-\mathrm{C} 23$ & & 4.73 & 8.63 \\
\hline 2-meC23 & & 6.36 & 5.00 \\
\hline 3-meC23 & & 2.95 & 2.99 \\
\hline$n-\mathrm{C} 24$ & & 0.77 & 0.82 \\
\hline $\mathrm{C} 25: 2$ & 24.30 & 0.29 & 0.48 \\
\hline $\mathrm{C} 25: 2 ; 2-\mathrm{meC}^{2} 4^{c}$ & 24.60 & 4.04 & 3.59 \\
\hline $\mathrm{C} 25: 1$ & 24.70 & 1.42 & 1.20 \\
\hline$n-\mathrm{C} 25$ & & 8.29 & 8.56 \\
\hline $\mathrm{C} 26: 2$ & 25.30 & 1.32 & 1.41 \\
\hline $2-\mathrm{meC} 25$ & & 0.24 & 0.28 \\
\hline $\mathrm{C} 26: 2 ; 3-\mathrm{meC} 25^{c}$ & 25.65 & 1.00 & 0.54 \\
\hline$n-\mathrm{C} 26$ & & 1.52 & 0.76 \\
\hline $\mathrm{C} 27: 2$ & 26.30 & 27.79 & 31.63 \\
\hline $\mathrm{C} 27: 2 ; \mathrm{C} 27: 1 ; 2-\mathrm{meC}^{2} 6^{c}$ & 26.60 & 0.78 & 0.79 \\
\hline $\mathrm{C} 27: 1$ & 26.70 & 3.30 & 1.20 \\
\hline Unknown & & 0.92 & 0.20 \\
\hline$n-\mathrm{C} 27$ & & 6.69 & 3.58 \\
\hline $11-\mathrm{meC} 27 ; \mathrm{C} 28: 2^{c}$ & 27.30 & 0.36 & 0.34 \\
\hline $2-\mathrm{meC} 27$ & & 0.18 & 0.14 \\
\hline 3-meC27 & & 0.11 & 0.10 \\
\hline$n-\mathrm{C} 28$ & & 0.31 & 0.12 \\
\hline $\mathrm{C} 29: 2$ & 28.30 & 0.20 & 0.12 \\
\hline$n-\mathrm{C} 29$ & & 2.53 & 0.32 \\
\hline$n-\mathrm{C} 31$ & & 0.00 & 0.19 \\
\hline$n-\mathrm{C} 33$ & & 0.00 & 0.24 \\
\hline$n-\mathrm{C} 34$ & & 0.00 & 0.18 \\
\hline$n-\mathrm{C} 35$ & & 0.00 & 0.30 \\
\hline$n-\mathrm{C} 36$ & & 0.00 & 0.30 \\
\hline$n-\mathrm{C} 37$ & & 0.00 & 0.31 \\
\hline $15,19-; 13,17-\operatorname{dimeC} 37^{c}$ & & 0.00 & 0.48 \\
\hline$n-\mathrm{C} 38$ & & 0.00 & 0.36 \\
\hline $\mathrm{C} 39: 2$ & & 0.05 & 0.00 \\
\hline$n-\mathrm{C} 39$ & & 0.00 & 0.35 \\
\hline$n-\mathrm{C} 40$ & & 0.00 & 0.25 \\
\hline $\mathrm{C} 41: 2$ & & 0.12 & 0.23 \\
\hline 13,23-dimeC41 & & 0.00 & 0.58 \\
\hline $\mathrm{C} 42: 2$ & & 0.23 & 0.00 \\
\hline $\mathrm{C} 43: 3$ & & 1.74 & 1.53 \\
\hline $\mathrm{C} 43: 2$ & & 8.23 & 7.10 \\
\hline C43:1 & & 1.52 & 1.79 \\
\hline $\mathrm{C} 44: 3$ & & 0.25 & 0.43 \\
\hline $\mathrm{C} 44: 2$ & & 0.38 & 0.49 \\
\hline $\mathrm{C} 45: 4$ & & 0.64 & 0.42 \\
\hline $\mathrm{C} 45: 3$ & & 5.10 & 5.78 \\
\hline $\mathrm{C} 45: 2$ & & 4.00 & 4.51 \\
\hline
\end{tabular}

${ }^{a}$ Percent of total hydrocarbon composition.

${ }^{b}$ Equivalent chain length.

${ }^{c}$ An isomeric mixture or two or more compounds coelute in this peak. 
TAble 3. Relative Abundance ${ }^{a}$ OF Hydrocarbons from Pseudergates AND FECAL PELlets of Cryptotermes brevis (WALKER)

\begin{tabular}{|c|c|c|}
\hline Hydrocarbons & Termites & Fecal pellets \\
\hline$n-\mathrm{C} 23$ & 0.08 & 0.17 \\
\hline 2-meC23 & 0.58 & 0.12 \\
\hline 3-meC23 & 1.87 & 0.46 \\
\hline$n-\mathrm{C} 24$ & 0.66 & 0.59 \\
\hline Unknown & 1.42 & 0.00 \\
\hline Unknown & 0.13 & 0.00 \\
\hline 2-meC24 & 4.20 & 1.05 \\
\hline 3-meC24 & 0.52 & 0.07 \\
\hline$n-\mathrm{C} 25$ & 12.48 & 11.76 \\
\hline $13-; 11-; 9-\mathrm{meC} 25^{b}$ & 0.64 & 0.24 \\
\hline $2-\mathrm{meC} 25$ & 1.00 & 0.34 \\
\hline 3-meC25 & 6.47 & 2.19 \\
\hline$n-\mathrm{C} 26$ & 1.76 & 1.65 \\
\hline $3,13-; 3,15$-dimeC $25^{b}$ & 0.14 & 0.10 \\
\hline 2-meC26 & 0.87 & 0.35 \\
\hline 3-meC26 & 0.15 & 0.00 \\
\hline$n-\mathrm{C} 27$ & 11.19 & 10.18 \\
\hline $13-; 11-\mathrm{meC} 27^{b}$ & 0.15 & 0.08 \\
\hline 2-meC27 & 0.10 & 0.06 \\
\hline 3 -meC27 & 0.69 & 0.28 \\
\hline$n-\mathrm{C} 28$ & 0.78 & 0.69 \\
\hline $\mathrm{C} 29: 1$ & 0.12 & 0.00 \\
\hline C29:1 & 0.06 & 0.00 \\
\hline$n-\mathrm{C} 29$ & 2.34 & 1.93 \\
\hline $13-\mathrm{meC} 29$ & 0.06 & 0.00 \\
\hline $2-\mathrm{meC} 29$ & 0.15 & 0.13 \\
\hline C31:1 & 0.27 & 0.11 \\
\hline$n-\mathrm{C} 31$ & 0.13 & 0.13 \\
\hline 3,7-dimeC31 & 0.14 & 0.06 \\
\hline $\mathrm{C} 33: 2$ & 0.13 & 0.00 \\
\hline C33:1 & 0.16 & 0.09 \\
\hline $\mathrm{C} 35: 3$ & 0.19 & 0.00 \\
\hline $\mathrm{C} 35: 2$ & 1.02 & 0.22 \\
\hline C35:1 & 0.23 & 0.00 \\
\hline C36:2 & 0.60 & 0.00 \\
\hline $\mathrm{C} 37: 3$ & 0.59 & 0.00 \\
\hline C $37: 2$ & 7.13 & 2.85 \\
\hline C37:1 & 1.35 & 0.64 \\
\hline C $38: 3$ & 0.28 & 0.00 \\
\hline C38:2 & 1.39 & 0.77 \\
\hline C38:1 & 0.22 & 0.00 \\
\hline C39:3 & 2.28 & 0.93 \\
\hline C39:2 & 9.14 & 9.78 \\
\hline C39:1 & 2.24 & 3.56 \\
\hline $\mathrm{C} 40: 3$ & 0.53 & 0.58 \\
\hline
\end{tabular}


TABLE 3. CONTINUED

\begin{tabular}{lcc}
\hline \multicolumn{1}{c}{ Hydrocarbons } & Termites & Fecal pellets \\
\hline $\mathrm{C} 40: 2 ; 15-; 13-\mathrm{meC}^{\prime} 9^{b}$ & 1.13 & 2.06 \\
$\mathrm{C} 40: 1$ & 0.21 & 0.40 \\
$\mathrm{C} 41: 3$ & 3.60 & 6.18 \\
$\mathrm{C} 41: 2$ & 4.29 & 10.93 \\
$\mathrm{C} 41: 1$ & 1.43 & 3.98 \\
$\mathrm{C} 42: 3$ & 0.35 & 0.71 \\
$\mathrm{C} 42: 2 ; 15-; 13-\mathrm{meC}^{\prime} 1^{b}$ & 0.56 & 1.65 \\
$\mathrm{C} 42: 1$ & 0.18 & 0.17 \\
$\mathrm{C} 43: 3$ & 2.03 & 4.45 \\
$\mathrm{C} 43: 2$ & 2.32 & 6.44 \\
$\mathrm{C} 43: 1$ & 1.90 & 3.62 \\
$\mathrm{C} 44: 2 ; 15-; 13-\mathrm{meC}^{2} 3^{b}$ & 0.27 & 1.38 \\
$\mathrm{C} 45: 3$ & 2.22 & 3.33 \\
$\mathrm{C} 45: 2$ & 2.06 & 2.51 \\
$\mathrm{C} 45: 1$ & 0.80 & 0.00 \\
\hline
\end{tabular}

${ }^{a}$ Percent of total hydrocarbon composition.

${ }^{b} \mathrm{An}$ isomeric mixture or two or more compounds coelute in this peak.

Hydrocarbons occurred in two groups: the early eluting compounds were dominated by one olefin, $\mathrm{C} 27: 1$; late-eluting compounds were predominantly olefins (Figure 4, Table 4). $n$-Alkanes comprised $16 \%$ of the total hydrocarbon. Alkenes, alkadienes, and alkatrienes were the predominant class of cuticular hydrocarbons, representing $70.3 \%$ of the total hydrocarbon; C27:1 accounts for $28 \%$ of the total hydrocarbon. Terminally branched monomethylalkanes amounted to $11.1 \%$ and internally branched monomethylalkanes $2.8 \%$ of the total hydrocarbon. No di- or trimethylalkanes were detected.

I. minor has an extensive geographical and ecological range. It is common in structures and in trees, especially at pruning scars, in the southwestern, coastal counties of California. Its distribution extends north to Mendocino County, south into Baja California and along the coast of western Mexico, and east into eastern Arizona and Utah (Weesner, 1970). I. minor has been introduced, and possibly established, in two separate locations on the island of Oahu (Grace et al., 2002). The general pattern of the chromatograms of I. minor (Figure 5) was more similar to that of M. hubbardi (see below) than that of I. immigrans (Figure 2), or other drywood termites from the tropics (Haverty et al., 1997, 2000). Hydrocarbons occurred in a continuous "series" from $n$-C23 to 13,17-dime C43 without the unoccupied region from C29 to C33 (Table 5). $n$-Alkanes comprised $17.7 \%$ of the total hydrocarbon. The unsaturated components constituted only $19.7 \%$ of the total hydrocarbon, a proportion much lower than that of tropical kalotermitids. Late-eluting olefins predominated in 
TABle 4. Relative Abundance ${ }^{a}$ OF Hydrocarbons from Pseudergates AND FECAL PELLETS OF Cryptotermes cynocephalus LIGHT

\begin{tabular}{|c|c|c|c|}
\hline Hydrocarbons & $\mathrm{ECL}^{b}$ & Termites & Fecal pellets \\
\hline$n-\mathrm{C} 20$ & & 0.00 & 1.15 \\
\hline$n-\mathrm{C} 21$ & & 0.00 & 3.24 \\
\hline 2-meC21 & & 0.00 & 0.23 \\
\hline$n-\mathrm{C} 22$ & & 0.00 & 3.68 \\
\hline $2-\mathrm{meC} 22$ & & 0.00 & 0.45 \\
\hline 3-meC22 & & 0.00 & 0.31 \\
\hline$n-\mathrm{C} 23$ & & 0.39 & 3.95 \\
\hline 2-meC23 & & 0.40 & 0.61 \\
\hline 3-meC23 & & 0.00 & 0.46 \\
\hline$n-\mathrm{C} 24$ & & 0.20 & 1.58 \\
\hline 2-meC24 & & 3.65 & 2.70 \\
\hline 3-meC24 & & 0.00 & 0.42 \\
\hline$n-\mathrm{C} 25$ & & 9.17 & 13.91 \\
\hline $13-; 11-\mathrm{meC} 25^{c}$ & & 0.93 & 1.04 \\
\hline $2-\mathrm{meC} 25 ; \mathrm{C} 26: 1^{c}$ & 25.70 & 2.81 & 2.02 \\
\hline 3-meC25 & & 3.47 & 3.93 \\
\hline$n-\mathrm{C} 26$ & & 0.70 & 1.12 \\
\hline $\mathrm{C} 27: 1$ & 26.30 & 0.61 & 0.31 \\
\hline $\mathrm{C} 27: 1$ & 26.70 & 27.41 & 32.94 \\
\hline$n-\mathrm{C} 27$ & & 4.91 & 4.13 \\
\hline $\mathrm{C} 28: 1$ & 27.30 & 0.20 & 0.09 \\
\hline 13-; 11-; 9-meC27 & & 1.23 & 0.89 \\
\hline $2-\mathrm{meC} 27 ; \mathrm{C} 28: 1^{c}$ & 27.70 & 0.96 & 0.73 \\
\hline $3-\mathrm{meC} 27$ & & 0.46 & 0.26 \\
\hline$n-\mathrm{C} 28$ & & 0.11 & 0.30 \\
\hline $\mathrm{C} 29: 1$ & 28.30 & 0.32 & 0.15 \\
\hline $\mathrm{C} 29: 2$ & 28.50 & 0.36 & 0.18 \\
\hline $\mathrm{C} 29: 1$ & 28.70 & 0.92 & 0.67 \\
\hline$n-\mathrm{C} 29$ & & 0.44 & 0.51 \\
\hline $\mathrm{C} 29: 2$ & 29.30 & 0.24 & 0.06 \\
\hline 2-meC29 & & 0.39 & 0.11 \\
\hline $\mathrm{C} 31: 2$ & 30.50 & 1.21 & 0.20 \\
\hline C31:1 & 30.70 & 0.37 & 0.00 \\
\hline 2-meC30 & & 0.88 & 0.22 \\
\hline$n-\mathrm{C} 31$ & & 0.09 & 0.36 \\
\hline $\mathrm{C} 35: 2$ & 34.50 & 0.22 & 0.00 \\
\hline $\mathrm{C} 37: 2$ & 36.50 & 3.82 & 0.30 \\
\hline $\mathrm{C} 37: 1$ & 36.70 & 0.58 & 0.00 \\
\hline C38:2 & & 0.42 & 0.00 \\
\hline $\mathrm{C} 39: 3$ & & 0.85 & 0.00 \\
\hline C39:2 & & 4.69 & 0.62 \\
\hline C39:1 & & 0.53 & 0.00 \\
\hline $\mathrm{C} 40: 2$ & & 0.48 & 0.17 \\
\hline $\mathrm{C} 41: 3$ & & 0.91 & 0.09 \\
\hline $\mathrm{C} 41: 2$ & & 8.14 & 4.31 \\
\hline $\mathrm{C} 41: 1$ & & 4.04 & 2.09 \\
\hline $15-; 13-\mathrm{meC} 41 ; \mathrm{C} 42: 2^{c}$ & & 1.29 & 0.95 \\
\hline $\mathrm{C} 43: 3$ & & 1.22 & 0.92 \\
\hline $\mathrm{C} 43: 2$ & & 8.15 & 6.35 \\
\hline C43:1 & & 1.82 & 1.28 \\
\hline
\end{tabular}

${ }^{a}$ Percent of total hydrocarbon composition.

${ }^{b}$ Equivalent chain length.

${ }^{c}$ An isomeric mixture or two or more compounds coelute in this peak. 
TABle 5. Relative ABundancE ${ }^{a}$ OF Hydrocarbons From PseUdergates AND FECAL PELLETS OF Incisitermes minor (HAGEN)

\begin{tabular}{|c|c|c|}
\hline Hydrocarbons & Termites & Fecal pellets \\
\hline$n-\mathrm{C} 23$ & 1.37 & 1.55 \\
\hline 2-meC23 & 3.38 & 2.91 \\
\hline $3-\mathrm{meC} 23$ & 2.22 & 1.39 \\
\hline$n-\mathrm{C} 24$ & 0.42 & 0.78 \\
\hline$n-\mathrm{C} 25$ & 9.20 & 8.91 \\
\hline $2-\mathrm{meC} 25$ & 0.22 & 0.18 \\
\hline 3-meC25 & 0.60 & 0.36 \\
\hline$n-\mathrm{C} 26$ & 1.07 & 0.97 \\
\hline$n-\mathrm{C} 27$ & 4.86 & 4.35 \\
\hline $3-\mathrm{meC} 27$ & 0.25 & 0.22 \\
\hline$n-\mathrm{C} 29$ & 0.80 & 0.76 \\
\hline 9,13-dimeC29 & 0.94 & 0.55 \\
\hline 9,13,17-trimeC29 & 0.84 & 0.25 \\
\hline 10,14-dimeC30 & 1.37 & 0.69 \\
\hline $10,14,18$-trimeC30 & 1.05 & 0.49 \\
\hline $15-; 13-; 11-; 9-\mathrm{meC} 31^{b}$ & 0.32 & 0.00 \\
\hline $13,17-; 11,15$-dimeC $31^{b}$ & 12.99 & 8.17 \\
\hline $11,15,19-; 9,13,17$-trimeC $31^{b}$ & 1.68 & 1.13 \\
\hline 7,11,17-trimeC31 & 0.31 & 0.27 \\
\hline $14-; 12-\mathrm{meC} 32^{b}$ & 0.30 & 0.18 \\
\hline 12,16-dimeC32 & 3.54 & 2.98 \\
\hline $10,14,18-; 8, \mathrm{X}, \mathrm{X}$-trimeC $32^{b, c}$ & 0.46 & 0.49 \\
\hline $15-; 13-\mathrm{meC} 33$ & 1.28 & 0.53 \\
\hline $13,17-; 11,15-; 9,13-\operatorname{dimeC} 33^{b}$ & 8.66 & 8.80 \\
\hline $9,13,17-; 7, \mathrm{X}, \mathrm{X}$-trimeC $33^{b, c}$ & 1.28 & 1.97 \\
\hline $\mathrm{C} 35: 3$ & 1.45 & 1.08 \\
\hline $\mathrm{C} 35: 2$ & 3.60 & 3.32 \\
\hline 12,16-dimeC34 & 1.30 & 2.03 \\
\hline $15-; 13-; 11-\mathrm{meC} 35^{b}$ & 0.82 & 0.77 \\
\hline 13,17-dimeC35 & 2.67 & 4.68 \\
\hline 9,13,17-trimeC35 & 0.50 & 1.91 \\
\hline $\mathrm{C} 37: 3$ & 4.30 & 5.37 \\
\hline $\mathrm{C} 37: 3$ & 1.73 & 2.56 \\
\hline $\mathrm{C} 37: 2$ & 2.30 & 1.88 \\
\hline 12,16-dimeC36 & 0.32 & 0.80 \\
\hline $\mathrm{C} 37: 1$ & 0.28 & 0.52 \\
\hline $10,14,18$-trimeC 36 & 0.28 & 0.41 \\
\hline $13 ; 11-\mathrm{meC} 37^{b}$ & 0.67 & 0.59 \\
\hline $13,17-; 11,15$-dimeC $37^{b}$ & 1.75 & 1.92 \\
\hline 9,13,17-trimeC37 & 0.27 & 0.58 \\
\hline $\mathrm{C} 39: 3$ & 1.76 & 5.43 \\
\hline C39:3; C39:2; 12,16-dimeC38 $8^{b}$ & 0.73 & 1.16 \\
\hline $\mathrm{C} 39: 1$ & 1.13 & 1.16 \\
\hline $13-; 11-\mathrm{meC} 39^{b}$ & 0.96 & 0.89 \\
\hline $13,17-; 11,15$-dimeC $39^{b}$ & 0.99 & 0.97 \\
\hline
\end{tabular}


TABLE 5. CONTINUED

\begin{tabular}{|c|c|c|}
\hline Hydrocarbons & Termites & Fecal pellets \\
\hline $12-; 10-\mathrm{meC} 40^{b}$ & 0.30 & 0.44 \\
\hline 12,16-dimeC40 & 0.40 & 0.40 \\
\hline C41:1 & 1.51 & 1.65 \\
\hline $13-; 11-\mathrm{meC} 41^{b}$ & 1.71 & 1.28 \\
\hline $13,17-; 11,15$-dimeC $41^{b}$ & 4.46 & 4.69 \\
\hline 9,13,17-trimeC41 & 0.36 & 0.74 \\
\hline $12-; 10-\mathrm{meC} 42^{b}$ & 0.25 & 0.35 \\
\hline 12,16-dimeC42 & 0.63 & 0.62 \\
\hline $\mathrm{C} 43: 1$ & 1.62 & 1.36 \\
\hline $15-; 13-\mathrm{meC} 43^{b}$ & 0.30 & 0.32 \\
\hline 13,17 -dimeC43 ${ }^{b}$ & 1.21 & 1.24 \\
\hline
\end{tabular}

${ }^{a}$ Percent of total hydrocarbon composition.

${ }^{b}$ An isomeric mixture or two or more compounds coelute in this peak.

${ }^{c}$ The exact methyl branch positions are uncertain.

this component. The terminally branched monomethylalkanes amounted to $6.7 \%$ of the total hydrocarbon, whereas internally branched monomethylalkanes totaled $6.9 \%$. Dimethylalkanes were the predominant class of compounds, making up $42 \%$ of the total hydrocarbon. I. minor also made a homologous series of trimethylalkanes representing $7.0 \%$ of the total hydrocarbon.

P. corniceps is found throughout most of the islands of the West Indies (Scheffrahn et al., 1994; Haverty et al., 1997). It can be found in dead wood of native vegetation, including fence posts, but has not yet been recorded from structures (Collins et al., 1997). The cuticular hydrocarbon mixture of P. corniceps (Figure 6) was similar in gross comparison to that of C. brevis (Figure 3). The early eluting components were almost exclusively $n$-alkanes and terminally branched monomethylalkanes, and the late eluting compounds were primarily olefins (Table 6). $n$-Alkanes comprised $8.7 \%$ of the total hydrocarbon. As with $C$. brevis, the alkenes, alkadienes, and alkatrienes were the predominant class of cuticular hydrocarbons, totalling over $76 \%$ of the total hydrocarbon. The terminally branched monomethylalkanes made up $12.2 \%$ of the total hydrocarbon.

P. occidentis occurs primarily within the general limits of the Sonoran Desert in southern Arizona and Baja California. P. occidentis has been found in few tree species, most commonly in blue paloverde, Parkinsonia florida (Bentham ex A. Gray) S. Watson (Nutting, 1966). The cuticular hydrocarbon mixture of $P$. occidentis has never been published, therefore, we provide a detailed description here. Once again, cuticular hydrocarbons occurred in two distinct groups: early eluting ( 25 to 31 carbons in the parent chain) and lateeluting compounds (41 to 43 carbons in the parent chain). The early eluting 
TABle 6. Relative AbundancE ${ }^{a}$ OF Hydrocarbons From PseUdergates AND FECAL PELlets of Procryptotermes corniceps (SNYDER)

\begin{tabular}{|c|c|c|}
\hline Hydrocarbons & Termites & Fecal pellets \\
\hline 2-meC24 & 1.52 & 0.68 \\
\hline 3-meC24 & 0.06 & 0.00 \\
\hline$n-\mathrm{C} 25$ & 1.62 & 1.37 \\
\hline 2-meC25 & 1.87 & 0.55 \\
\hline $3-\mathrm{meC} 25$ & 3.10 & 1.58 \\
\hline$n-\mathrm{C} 26$ & 0.87 & 0.47 \\
\hline $2-\mathrm{meC} 26$ & 4.18 & 1.55 \\
\hline 3-meC26 & 0.27 & 0.00 \\
\hline$n-\mathrm{C} 27$ & 5.95 & 4.43 \\
\hline $2-\mathrm{meC} 27$ & 0.36 & 0.12 \\
\hline 3-meC27 & 0.70 & 0.15 \\
\hline$n-\mathrm{C} 28$ & 0.11 & 0.09 \\
\hline $2-\mathrm{meC} 28$ & 0.17 & 0.27 \\
\hline$n-\mathrm{C} 29$ & 0.18 & 0.00 \\
\hline $\mathrm{C} 35: 2$ & 0.09 & 0.00 \\
\hline $\mathrm{C} 37: 3$ & 0.84 & 0.41 \\
\hline $\mathrm{C} 37: 2$ & 1.65 & 1.02 \\
\hline C37:1 & 0.08 & 0.06 \\
\hline C38:3 & 0.14 & 0.00 \\
\hline $\mathrm{C} 38: 2$ & 0.65 & 0.43 \\
\hline C39:3 & 4.88 & 4.04 \\
\hline C39:2 & 8.10 & 8.63 \\
\hline C39:1 & 13.63 & 1.62 \\
\hline $\mathrm{C} 40: \mathrm{X}^{c}$ & 0.58 & 0.48 \\
\hline $\mathrm{C} 40: 3$ & 1.32 & 1.53 \\
\hline $\mathrm{C} 40: 2 ; 15-; 13-\mathrm{meC} 39^{b}$ & 3.53 & 3.31 \\
\hline $\mathrm{C} 41: 3$ & 13.35 & 22.83 \\
\hline $\mathrm{C} 41: 2$ & 15.11 & 20.27 \\
\hline $\mathrm{C} 41: 1$ & 0.96 & 1.23 \\
\hline C41:1 & 2.65 & 3.62 \\
\hline $\mathrm{C} 42: \mathrm{X}^{c}$ & 0.97 & 1.39 \\
\hline $\mathrm{C} 42: 3$ & 1.46 & 2.24 \\
\hline $15-; 13-\mathrm{meC} 41^{b}$ & 1.26 & 1.68 \\
\hline $\mathrm{C} 42: 2$ & 0.82 & 1.03 \\
\hline $\mathrm{C} 43: 4$ & 0.80 & 1.47 \\
\hline $\mathrm{C} 43: 3$ & 4.04 & 8.08 \\
\hline $\mathrm{C} 43: 2$ & 1.79 & 3.09 \\
\hline C43:1 & 0.37 & 0.29 \\
\hline
\end{tabular}

${ }^{a}$ Percent of total hydrocarbon composition.

${ }^{b}$ An isomeric mixture or two or more compounds coelute in this peak.

${ }^{c}$ This peak appears to be a mixture of unsaturated compounds, their exact identities are undetermined. 

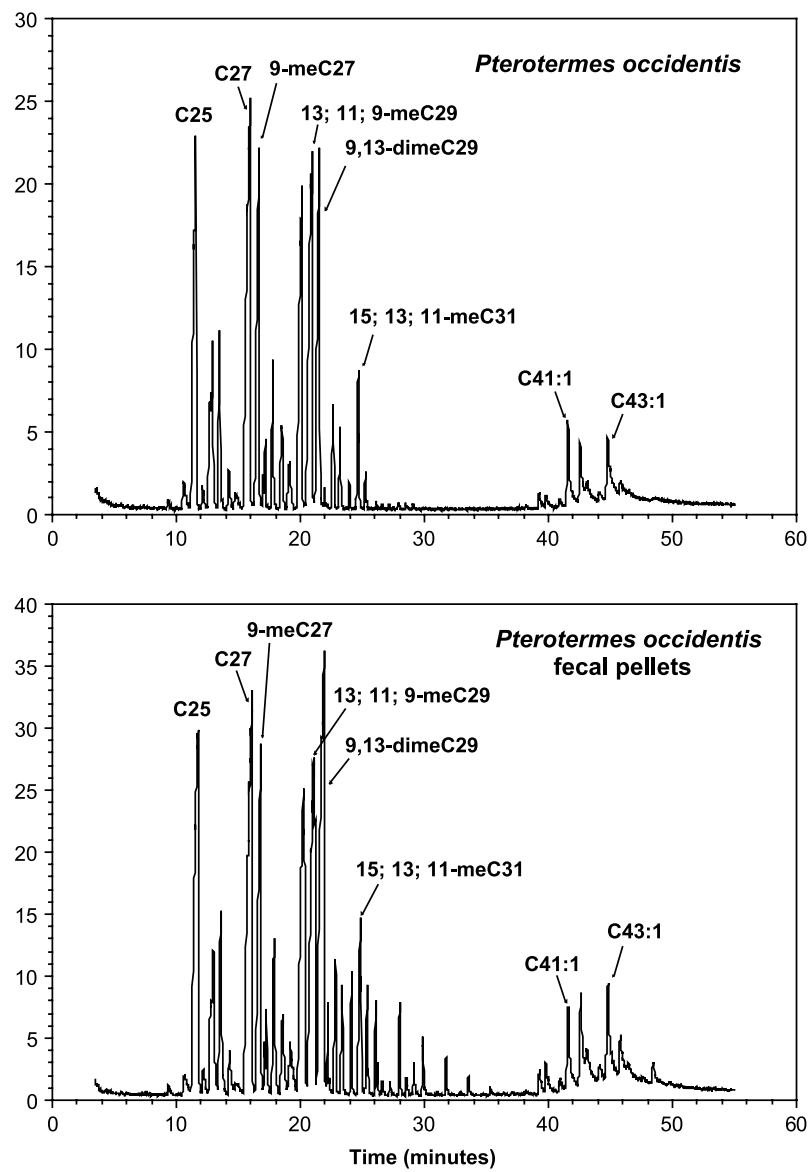

FIG. 7. Total ion chromatogram of the cuticular hydrocarbons from pseudergates of Pterotermes occidentis (Walker) from the Santa Rita Experimental Range near Tucson, $\mathrm{AZ}$, and fecal pellets from the same collection.

compounds predominated, representing over 92 percent of the total hydrocarbon (Figure 7, Table 7).

$n$-Alkanes were, by far, the most abundant hydrocarbons representing over $40 \%$ of the total (Table 7 ). $n$-C25, $n$-C27, and $n$-C29 comprised ca. $10.5 \%$, $14.7 \%$, and $9.1 \%$ of the total hydrocarbon, respectively. The other $n$-alkanes accounted for $6.6 \%$ of the total (Table 7). In contrast to the tropical drywood termite species, olefins were not the predominant hydrocarbons, representing only $5.0 \%$ of the total (Table 7). Two monoenes, C41:1 and C43:1, made up the majority of these hydrocarbons. 

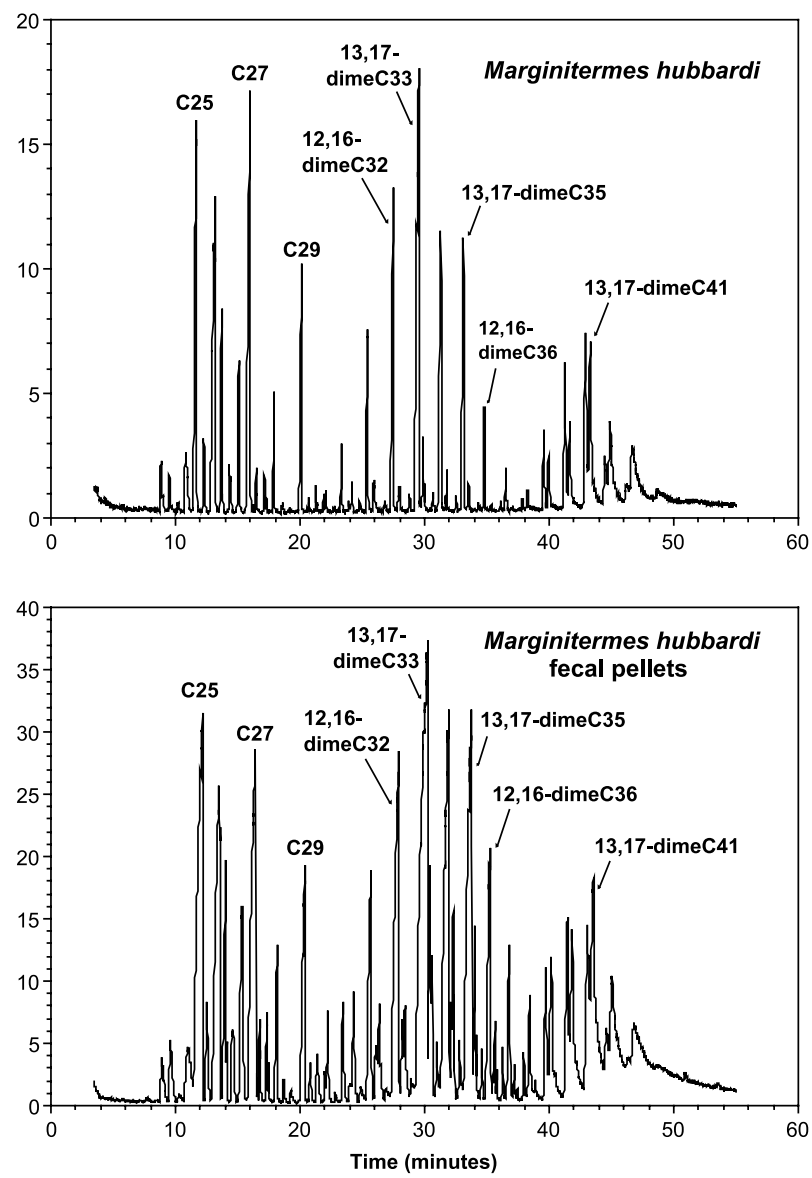

FIG. 8. Total ion chromatogram of cuticular hydrocarbons from pseudergates of Marginitermes hubbardi (Banks) from the Saguaro National Monument southeast of Tucson, AZ, and fecal pellets from the same collection.

Terminally branched monomethylalkanes were identified for $\mathrm{C} 24$ to $\mathrm{C} 27$ (Table 7). 2-MeC25 and $3-\mathrm{meC} 25$ accounted for $2.8 \%$ and $2.6 \%$ of the total hydrocarbon, respectively. The remainder of these terminally branched monomethylalkanes comprised only $2.2 \%$ of the total. Internally branched monomethylalkanes comprised $33.0 \%$ of the total hydrocarbon (Table 7). The homologous series of internally branched monomethylalkanes, C27, C29, C31, and $\mathrm{C} 41$ constituted $26.9 \%$ of the total hydrocarbon; none of the other internally branched monomethylalkanes represented more than $0.85 \%$ of the total. Dimethylalkanes comprised $13.4 \%$ of the total hydrocarbon; 9,13-dimeC29 
TABle 7. Relative AbUndance ${ }^{a}$ OF Hydrocarbons from Pseudergates AND FeCAl PELlets of Pterotermes occidentis (WALKER)

\begin{tabular}{|c|c|c|}
\hline Hydrocarbons & Termites & Fecal pellets \\
\hline$n-\mathrm{C} 24$ & 0.16 & 0.16 \\
\hline $2-\mathrm{meC} 24$ & 0.75 & 0.43 \\
\hline$n-\mathrm{C} 25$ & 10.52 & 9.17 \\
\hline $13-; 11-; 9-\mathrm{meC} 25^{b}$ & 0.51 & 0.38 \\
\hline 2-meC25 & 2.79 & 1.00 \\
\hline $3-\mathrm{meC} 25$ & 2.62 & 2.57 \\
\hline$n-\mathrm{C} 26$ & 3.39 & 2.90 \\
\hline $9-; 8-\mathrm{meC}_{2} 6^{b}$ & 0.85 & 0.72 \\
\hline 2-meC26 & 0.51 & 0.37 \\
\hline$n-\mathrm{C} 27$ & 14.71 & 11.97 \\
\hline $13-; 11-; 9-\operatorname{meC} 27^{b}$ & 9.59 & 7.39 \\
\hline 9,13-dime; 2 -meC $27^{b}$ & 0.47 & 0.41 \\
\hline $3-\mathrm{meC} 27$ & 0.73 & 0.73 \\
\hline$n-\mathrm{C} 28$ & 2.32 & 2.26 \\
\hline $12-; 10-; 9 ; 8-\mathrm{meC}_{2} 8^{b}$ & 2.03 & 1.54 \\
\hline $9,13-; 8,12-; 8,14-\operatorname{dimeC} 28^{b}$ & 1.40 & 1.32 \\
\hline$n-\mathrm{C} 29$ & 9.13 & 9.37 \\
\hline 15-; 13-; 11-meC29 $9^{b}$ & 13.17 & 10.55 \\
\hline 9,13-dimeC29 & 9.63 & 12.61 \\
\hline$n-\mathrm{C} 30$ & 0.19 & 0.56 \\
\hline $14-; 12-; 10-\mathrm{meC}^{2} 0^{b}$ & 1.81 & 1.84 \\
\hline $\mathrm{C} 31: 1 ; 10,14-\operatorname{dimeC} 30^{b}$ & 1.25 & 1.28 \\
\hline$n-\mathrm{C} 31$ & 0.33 & 1.01 \\
\hline $15-; 13-; 11-; 9-\operatorname{meC} 31^{b}$ & 2.52 & 2.81 \\
\hline 9,13-dimeC31 & 0.37 & 0.89 \\
\hline 7,13-dimeC31 & 0.11 & 0.15 \\
\hline$n$-C32 & 0.07 & 0.74 \\
\hline 3,7-dimeC31 & 0.00 & 0.21 \\
\hline $14-; 12-; 10-\mathrm{meC} 32^{b}$ & 0.08 & 0.19 \\
\hline 9,13-dimeC32 & 0.06 & 0.18 \\
\hline$n-\mathrm{C} 33$ & 0.12 & 0.84 \\
\hline $13-; 11-; 9-\mathrm{meC}_{3} 3^{b}$ & 0.09 & 0.23 \\
\hline 9,13-dimeC33 & 0.06 & 0.33 \\
\hline$n$-C34 & 0.00 & 0.49 \\
\hline$n-\mathrm{C} 35$ & 0.00 & 0.35 \\
\hline$n-\mathrm{C} 36$ & 0.00 & 0.17 \\
\hline$n-\mathrm{C} 37$ & 0.00 & 0.11 \\
\hline $13-; 11-\mathrm{meC} 39^{b}$ & 0.35 & 0.42 \\
\hline 9,13-dimeC39 & 0.33 & 0.64 \\
\hline $14-; 13-; 12-\mathrm{meC} 40^{b}$ & 0.14 & 0.28 \\
\hline C41:1 & 2.13 & 1.84 \\
\hline 15-; 13-; 11-meC41 ${ }^{b}$ & 1.57 & 2.00 \\
\hline 9,13-dimeC41 & 0.63 & 1.34 \\
\hline $14-; 13-; 12-; 11-\mathrm{meC} 42^{b}$ & 0.17 & 0.71 \\
\hline C43:1 & 2.20 & 3.03 \\
\hline $15-; 13-; 11-\mathrm{meC} 43^{b}$ & 0.17 & 1.51 \\
\hline
\end{tabular}

${ }^{a}$ Percent of total hydrocarbon composition.

${ }^{b} \mathrm{An}$ isomeric mixture or two or more compounds coelute in this peak. 
TABle 8. Relative ABundancE ${ }^{a}$ OF Hydrocarbons From PseUdergates AND FECAL PELLETS OF Marginitermes hubbardi (BANKS)

\begin{tabular}{|c|c|c|}
\hline Hydrocarbons & Termites & Fecal Pellets \\
\hline$n-\mathrm{C} 24$ & 0.16 & 0.16 \\
\hline 2-meC24 & 0.75 & 0.43 \\
\hline$n-\mathrm{C} 25$ & 10.52 & 9.17 \\
\hline $13-; 11-; 9-\mathrm{meC}^{2} 5^{b}$ & 0.51 & 0.38 \\
\hline $2-\mathrm{meC} 25$ & 2.79 & 1.00 \\
\hline $3-\mathrm{meC} 25$ & 2.62 & 2.57 \\
\hline$n-\mathrm{C} 26$ & 3.39 & 2.90 \\
\hline $9-; 8-\mathrm{meC}_{2} 6^{b}$ & 0.85 & 0.72 \\
\hline 2-meC26 & 0.51 & 0.37 \\
\hline$n-\mathrm{C} 27$ & 14.71 & 11.97 \\
\hline $13-; 11-; 9-\mathrm{meC} 27^{b}$ & 9.59 & 7.39 \\
\hline 9,13-dime; 2 -meC $27^{b}$ & 0.47 & 0.41 \\
\hline 3-meC27 & 0.73 & 0.73 \\
\hline$n-\mathrm{C} 28$ & 2.32 & 2.26 \\
\hline $12-; 10-; 9-; 8-\mathrm{meC}^{2} 8^{b}$ & 2.03 & 1.54 \\
\hline $9,13-; 8,12-; 8,14-\operatorname{dimeC} 28^{b}$ & 1.40 & 1.32 \\
\hline$n-\mathrm{C} 29$ & 9.13 & 9.37 \\
\hline $15-; 13-; 11-\mathrm{meC} 29^{b}$ & 13.17 & 10.55 \\
\hline 9,13-dimeC29 & 9.63 & 12.61 \\
\hline$n-\mathrm{C} 30$ & 0.19 & 0.56 \\
\hline $14-; 12-; 10-\mathrm{meC} 30^{b}$ & 1.81 & 1.84 \\
\hline $\mathrm{C} 31: 1 ; 10,14$-dimeC $30^{b}$ & 1.25 & 1.28 \\
\hline$n-\mathrm{C} 31$ & 0.33 & 1.01 \\
\hline $15-; 13-; 11-; 9-\operatorname{meC} 31^{b}$ & 2.52 & 2.81 \\
\hline 9,13-dimeC31 & 0.37 & 0.89 \\
\hline 7,13-dimeC31 & 0.11 & 0.15 \\
\hline$n-\mathrm{C} 32$ & 0.07 & 0.74 \\
\hline 3,7-dimeC31 & 0.00 & 0.21 \\
\hline $14-; 12-; 10-\mathrm{meC} 32^{b}$ & 0.08 & 0.19 \\
\hline 9,13-dimeC32 & 0.06 & 0.18 \\
\hline$n-\mathrm{C} 33$ & 0.12 & 0.84 \\
\hline $13-; 11-; 9-\operatorname{meC}^{3} 3^{b}$ & 0.09 & 0.23 \\
\hline 9,13-dimeC33 & 0.06 & 0.33 \\
\hline$n-\mathrm{C} 34$ & 0.00 & 0.49 \\
\hline$n-\mathrm{C} 35$ & 0.00 & 0.35 \\
\hline$n-\mathrm{C} 36$ & 0.00 & 0.17 \\
\hline$n-\mathrm{C} 37$ & 0.00 & 0.11 \\
\hline $13-; 11-\mathrm{meC} 39^{b}$ & 0.35 & 0.42 \\
\hline 9,13-dimeC39 & 0.33 & 0.64 \\
\hline $14-; 13-; 12-\mathrm{meC} 40^{b}$ & 0.14 & 0.28 \\
\hline $\mathrm{C} 41: 1$ & 2.13 & 1.84 \\
\hline $15-; 13-; 11-\mathrm{meC} 41^{b}$ & 1.57 & 2.00 \\
\hline 9,13-dimeC41 & 0.63 & 1.34 \\
\hline $14-; 13-; 12-; 11-\mathrm{meC} 42^{b}$ & 0.17 & 0.71 \\
\hline C43:1 & 2.20 & 3.03 \\
\hline $15-; 13-; 11-\mathrm{meC} 43^{b}$ & 0.17 & 1.51 \\
\hline
\end{tabular}

${ }^{a}$ Percent of total hydrocarbon composition.

${ }^{b} \mathrm{An}$ isomeric mixture or two or more compounds coelute in this peak. 

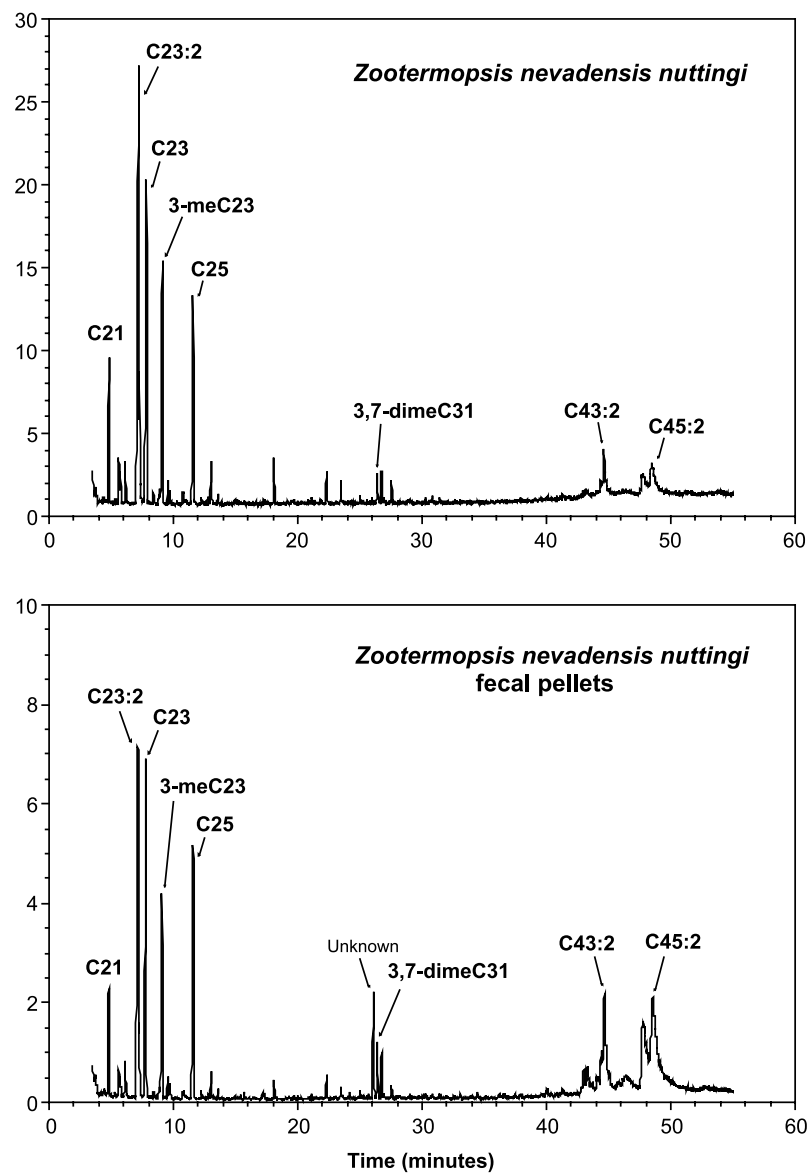

FIG. 9. Total ion chromatogram of cuticular hydrocarbons from pseudergates of Zootermopsis nevadensis nuttingi Haverty and Thorne from the Klamath National Forest northeast of Mount Shasta, CA, and fecal pellets from the same collection.

was the foremost member of this class with $9.6 \%$ of the total (Table 7 ). No trimethylalkanes were detected (Table 7).

M. hubbardi is one of the most common drywood termites in the Sonoran Desert. It is often found in the skeletal remains of the saguaro cactus, Cereus giganteus Englm., and is also commonly found in wood of structures. $M$. hubbardi replaces, or is more frequently encountered than, I. minor at lower elevations and drier regions of the Sonoran Desert (Weesner, 1970). As observed for $P$. occidentis, the cuticular hydrocarbon mixture of $M$. hubbardi has not been published, therefore, we provide a detailed description here. 
TABle 9. Relative ABUndancE ${ }^{a}$ OF Hydrocarbons From PSEUdergates AND FECAL PELlETS OF Zootermopsis nevadensis nuttingi HAVERTY AND THORNE

\begin{tabular}{|c|c|c|c|}
\hline Hydrocarbons & $\mathrm{ECL}^{b}$ & Termites & Fecal pellets \\
\hline$n-\mathrm{C} 21$ & & 5.15 & 3.75 \\
\hline 2-meC21 & & 1.80 & 0.92 \\
\hline 3-meC21 & & 1.32 & 0.64 \\
\hline$n-\mathrm{C} 22$ & & 1.71 & 1.15 \\
\hline $\mathrm{C} 23: 2$ & 22.50 & 29.21 & 16.10 \\
\hline $\mathrm{C} 23: 1$ & 22.70 & 3.21 & 1.87 \\
\hline$n-\mathrm{C} 23$ & & 15.34 & 12.58 \\
\hline $11-\mathrm{meC} 23$ & & 0.60 & 0.49 \\
\hline $2-\mathrm{meC} 23$ & & 0.73 & 0.41 \\
\hline $3-\mathrm{meC} 23$ & & 10.47 & 6.82 \\
\hline$n-\mathrm{C} 24$ & & 0.75 & 0.69 \\
\hline 3,7-dimeC23 & & 0.64 & 0.49 \\
\hline $\mathrm{C} 25: 1$ & 24.70 & 0.69 & 0.32 \\
\hline$n-\mathrm{C} 25$ & & 9.06 & 9.10 \\
\hline $11-\mathrm{meC} 25$ & & 0.00 & 0.28 \\
\hline $3-\mathrm{meC} 25$ & & 1.62 & 0.89 \\
\hline$n-\mathrm{C} 26$ & & 0.24 & 0.36 \\
\hline$n-\mathrm{C} 27$ & & 0.00 & 0.19 \\
\hline 3,7-dimeC27 & & 1.70 & 0.57 \\
\hline 3,7-dimeC29 & & 1.37 & 0.81 \\
\hline $\mathrm{C} 31: 2$ & 30.50 & 0.86 & 0.45 \\
\hline $\mathrm{C} 31: 1$ & 30.70 & 0.00 & 0.23 \\
\hline 7-meC31 & & 0.00 & 0.24 \\
\hline Unknown & & 0.00 & 4.10 \\
\hline 3,7-dimeC 31 & & 1.32 & 1.78 \\
\hline $\mathrm{C} 33: 3$ & & 1.31 & 1.32 \\
\hline C33:2 & & 0.89 & 0.51 \\
\hline $\mathrm{C} 43: \mathrm{X}^{c}$ & & 0.00 & 1.11 \\
\hline $\mathrm{C} 43: \mathrm{X}^{c}$ & & 0.00 & 2.21 \\
\hline $\mathrm{C} 43: \mathrm{X}^{c}$ & & 0.00 & 0.42 \\
\hline $\mathrm{C} 43: \mathrm{X}^{c}$ & & 0.00 & 1.01 \\
\hline $\mathrm{C} 43: 3$ & & 0.67 & 2.06 \\
\hline C43:3 & & 4.66 & 7.18 \\
\hline $\mathrm{C} 45: \mathrm{X}^{c}$ & & 1.63 & 9.20 \\
\hline $\mathrm{C} 45: 3$ & & 3.04 & 9.74 \\
\hline
\end{tabular}

${ }^{a}$ Percent of total hydrocarbon composition.

${ }^{b}$ Equivalent chain length.

${ }^{c}$ This peak appears to be a mixture of unsaturated compounds, their exact identities are undetermined. 
The general pattern of the chromatograms of M. hubbardi (Figure 8) was more similar to that of I. minor (Figure 5) than that of other drywood termites from Hawaii or the dry tropics (Figures 1-4, 6). Hydrocarbons occurred continuously from $n$-C25 to dimeC43 without the unoccupied region from $\mathrm{C} 29$ to C33. $n$-Alkanes present were $n$-C24, n-C25, n-C26, n-C27, n-C28, n-C29 $n$-C 30 , and $n$-C31 (Table 8). $n$-C25 and $n$-C27 were the most abundant, comprising $7.8 \%$ and $9.7 \%$ of the total hydrocarbon, respectively (Figure 8). The remaining $n$-alkanes amounted to $8.1 \%$ of the total hydrocarbon. There were no unsaturated components detected.

Terminally branched monomethylalkanes were identified for C23 to C27; 3 -meC25, representing $2.3 \%$ of the hydrocarbons, was the most abundant. Terminally branched monomethylalkanes constituted only $3.6 \%$ of the total hydrocarbon. Internally branched monomethylalkanes were the third most abundant class in M. hubbardi. Most were among the later-eluting compounds with carbon numbers in the parent chain ranging from 29 through 43 (Table 8). The isomeric mixture of 11-; 13-; 15-meC41 was the largest peak in this class accounting for $3.7 \%$ of the total (Figure 8 ).

In M. hubbardi, as in I. minor, dimethylalkanes were the predominant class of compounds, accounting for $53.9 \%$ of the hydrocarbon. The isomeric mixture of 11,15-; 13,17-dimeC33 was the most abundant peak accounting for $10.8 \%$ of the total hydrocarbon. M. hubbardi made a homologous series of internally branched dimethylalkanes $(9,13-; 11,15-; 12,16-; 13,17-)$ from C24 to C43 (Figure 8; Table 8). M. hubbardi also made numerous trimethylalkanes with 25 to 39 carbons in the parent chain; these trimethylalkanes accounted for $3.9 \%$ of the total hydrocarbon (Table 8).

Z. nevadensis nuttingi is the common dampwood termite found in the coastal forests of California, Oregon, and Washington west of the Cascade Range (Thorne et al., 1993). Zootermopsis species infest standing dead trees, stumps, and logs in contact with the soil. Like drywood termites, Zootermopsis species produce pelletized feces that are stored in chambers within their workings. Z. nevadensis nuttingi was originally designated Phenotype III of the North American Zootermopsis (Haverty et al., 1988). It was later designated as a subspecies of $Z$. nevadensis on the basis of its cuticular hydrocarbon mixture and its ability to recognize the other phenotype or subspecies, Z. nevadensis nevadensis, and react aggressively toward it (Haverty and Thorne, 1989). Morphologically, Z. n. nuttingi remains indistinguishable from Z. n. nevadensis (Thorne and Haverty, 1989). Their distributions are, for the most part, allopatric or parapatric (Thorne et al., 1993; Haverty et al., unpublished data).

The hydrocarbon mixture of $Z$. nevadensis nuttingi has been published previously (Haverty et al., 1988). This subspecies has since been more thoroughly characterized, and several compounds have been identified that were not previously reported. In particular, numerous olefins were identified by their mass 
spectra. These compounds can become unstable if not analyzed promptly after extraction, and this may account for their absence in the previous report (Haverty et al., 1988). Handling and extraction of specimens has been standardized to include drying of termites before extraction, thereby improving the recovery of hydrocarbons, especially olefins, from the cuticle (Haverty et al., 1996b).

The general pattern of the chromatograms of $Z$. nevadensis nuttingi (Figure 8) was similar to that of $N$. connexus (Figure 1), where the great majority of the hydrocarbons have 21 to 31 carbons in the parent chain. $n$-Alkanes present were $n$-C21, $n$-C22, $n$-C23, $n$-C24, and $n$-C25 (Table 9). $n$-C23 and $n$-C25 were the most abundant, comprising $15.3 \%$ and $9.1 \%$ of the total hydrocarbon, respectively (Figure 9). The remaining $n$-alkanes comprised $7.9 \%$ of the hydrocarbons.

The unsaturated components constituted $46.2 \%$ of the total hydrocarbon. Olefins with 23,43 , and 45 carbons predominated among the olefins. C23:2, $\mathrm{C} 23: 1, \mathrm{C} 43: 3$, and $\mathrm{C} 45: 3$ accounted for $29.2 \%, 3.2 \%, 4.7 \%$, and $3.0 \%$ of the total hydrocarbon, respectively (Figure 9; Table 9). The remaining unsaturated components amounted to only $6.1 \%$ of the total hydrocarbons.

2- and 3-Methylalkanes were identified for $\mathrm{C} 21$ to $\mathrm{C} 25$, with 3-meC23, representing $10.5 \%$ of the total hydrocarbon, being the most abundant (Table 9). These terminally branched monomethylalkanes represented $15.9 \%$ of the total hydrocarbon. A small amount of the internally branched 11-meC23 was seen. Dimethylalkanes were represented by a homologous series of 3,7-dimeCXX (Table 9).

Hydrocarbons from Termite Fecal Pellets. In general, the hydrocarbons from whole-body extracts of drywood termites were represented qualitatively and quantitatively in extracts of fecal pellets from the same species. Extracts of fecal pellets were more similar to those of their producers than they were to extracts of any other termite species or their fecal pellets. Cluster analysis with all hydrocarbons, using Euclidean distance, consistently paired the hydrocarbon mixture from fecal pellets with the hydrocarbon mixture from termites of the species that produced the pellets (Figure 10).

The hydrocarbon mixtures from whole-body extracts and that of fecal pellets were not identical; minor differences did occur and were likely the result of one or more factors. For example, some termite/pellet pairs were not from the same colony or location ( $N$. connexus, I. immigrans, and C. brevis); thus, the minor qualitative and quantitative differences between termites and fecal pellets could reflect intraspecific variation. When certain hydrocarbons were found in the termites, but not in the fecal pellets, and vice versa, they often occurred in small quantities, usually less than $1.0 \%$ of the total hydrocarbon component (Tables 1-9). Therefore, the lack of certain hydrocarbons could also be a function of the concentration of the extracts.

Paraffins, a series of $n$-alkanes, were found in both termite and fecal pellet extracts, but long-chain $n$-alkanes were found only in extracts of termite fecal 


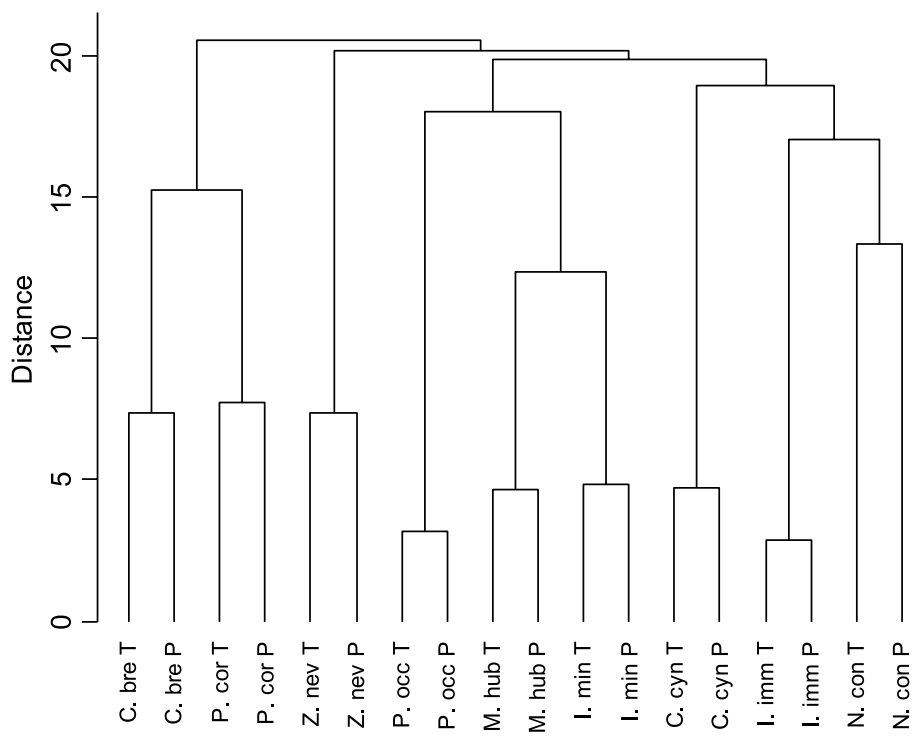

FIG. 10. Dendrogram from cluster analysis based on Euclidean distance of hydrocarbons extracted from eight species of drywood termites and one species of dampwood termite and their fecal pellets. N. con $=$ Neotermes connexus, I. imm $=$ Incisitermes immigrans, C. bre $=$ Cryptotermes brevis, C. cyn $=$ Cryptotermes cynocephalus, I. $\min =$ Incisitermes minor, $\mathrm{P}$. cor $=$ Procryptotermes corniceps, $\mathrm{P}$. occ $=$ Pterotermes occidentis, $\mathrm{M}$. hub $=$ Marginitermes hubbardi, and Z. nev = Zootermopsis nevadensis nuttingi.

pellets (Tables 1, 2, 7, 8; Figure 1). $n$-Alkanes with more than 31 carbon atoms are not usually seen in insects; insects apparently cannot synthesize $n$-alkanes containing more than 34 carbon atoms (Hadley, 1985). In plants, $n$-alkanes are the principal hydrocarbon fraction of the cuticlar lipids, ranging in length from 21 to 37 carbon atoms (Hadley, 1981). It is possible that the long-chain $n$-alkanes from drywood termite fecal pellets were a contaminant from plant origin, from the ubiquitous Parafilm ${ }^{\circledR}$ used in most laboratories, or even contamination from hand lotion. Such contamination complicated evaluation of chemotaxonomic characters of two species of Central American Nasutitermes (Howard et al., 1988).

Olefins comprised the majority of the hydrocarbon components in six of the nine termites discussed in this paper. Olefins are the least stable and most reactive of the cuticular hydrocarbons. However, for some reason, they remain stable in or on the cuticles of insects. For example, when hydrocarbons were extracted from 70-year-old museum specimens of scolytid cone beetles, Conophthorus ponderosae Hopkins, the mixtures were qualitatively identical to those collected and extracted by Page et al. (1990a). On the contrary, once 
extracted, cuticular hydrocarbons of Reticulitermes spp. containing mono-, diand trienes, lost much of the olefin component when dried and left in a vial for $24 \mathrm{hr}$, particularly the di- and trienes (Nelson and Haverty, unpublished observations). Thus, something prevents the oxidation of olefins while still in the cuticle. On or in fecal pellets, the olefins appeared to be as stable as they are within the cuticle of the termites.

The composition of the hydrocarbon mixture of insects is genetically controlled (Toolson and Kuper-Simbrón, 1989; Kaib et al., 1991; Page et al., 1991; Coyne et al., 1994). This composition can be slightly affected by diet and environmental conditions (Hadley, 1977; Espelie et al., 1994; Chapman et al., 1995; Howard, 1998; Woodrow et al., 2000). Because cuticular hydrocarbon mixtures are generally species-specific (see references in the Introduction), we did not expect to observe qualitative differences in cuticular hydrocarbon mixtures between recently collected $Z$. nevadensis nuttingi and those (Phenotype III) described by Haverty et al. (1988). Sevala et al. (2000) found similar qualitative differences between recently collected $Z$. nevadensis nevadensis and those (Phenotype I) reported by Haverty et al. (1988). In the present study, termites were extracted that had been dried, while Haverty et al. (1988) extracted freshly frozen (i.e., moist) termites. This could explain the difference, as drying termites first has been shown to enhance extraction of cuticular hydrocarbons, especially the late-eluting olefins (Haverty et al., 1996b). In addition, the olefin component of all four taxa reported by Haverty et al. (1988) was likely underreported, as samples were extracted, the extract dried under nitrogen, then sent to the University of Nevada for identification by GC-MS. Therefore, many (or all) of the olefins could have oxidized and no longer been present in the sample for identification. Furthermore, the equipment used by Haverty et al. (1988) (Finnigan 4023 mass spectrometer) was less sensitive than that used by Sevala et al. (2000) and in this study (HP5890 GC coupled with an HP5989 or 5970 MS, respectively). Thus, minor components and coeluting isomers could have been missed by Haverty et al. (1988).

From where do the hydrocarbons in drywood termite fecal pellets come? The answer to this question could be contamination from the cuticles of the termites moving within the gallery system strewn with fecal pellets. Three other scenarios are more likely, however. First, controlled cannibalism is a key to the nitrogen economy of a termite colony. Exuviae, injured or dead individuals, and excess members of any caste are commonly eaten (Moore, 1969). The hydrocarbons ingested could pass through the gut undigested, only to be compacted in the fecal pellets, although this has not yet been tested. Second, the termites moving and storing fecal pellets could secret a mixture of hydrocarbons from the various glands (labral, mandibular, labial, or salivary) associated with the mouthparts (Noirot, 1969). Third, and most likely, is that hydrocarbons are deposited onto the fecal pellets within the rectum during or after the fecal dehydration 
process. Oenocytes transmit hydrocarbons within the hemolymph to their epicuticular destinations (Schal et al., 1998; Sevala et al., 2000). Because the rectum is lined with cuticle (Child, 1934), hydrocarbons are likely transferred to the epicuticular cells of the rectum and then transferred to the surface, or lining.

What is the significance of fecal hydrocarbons that are similar to those found in termite cuticles? With more sampling across additional taxa, diagnostic hydrocarbons could be identified (Haverty et al., 1997) and a key to species developed (Haverty et al., 2000) for either nondiagnostic castes, such as larvae, nymphs, and pseudergates or workers, or even fecal pellets alone. This knowledge could potentially have applications for quarantine operations to identify species or damage to wood products or solid wood packing material and contain the spread of invasive termite species. In addition, our findings open up the possibility of other chemicals, such as semiochemicals, being deposited on or within fecal pellets with the pellets functioning as a slow-release mechanism (Souto and Kitayama, 2000). The possibility also exists that other wood-destroying insects could also be identified to species according to the hydrocarbons in their feces.

Acknowledgments-The authors thank C.H.M. Tome, R.J. Oshiro, S.Y. Uchima, and T.K. Brown for technical assistance and J.A. Baldwin for assistance with the cluster analysis. We extend thanks to Dr. and Mrs. H. Jarecki, J. Lazell, and the staff of the Guana Island Club for support and hospitality during the portion of this project conducted in the British Virgin Islands. This research was made possible, in part, by a grant to MIH by the Conservation Agency (through grants from the Falconwood Foundation), by a cooperative agreement between the University of Hawaii and the Pacific Southwest Research Station, by McIntire-Stennis funds for forestry research (J.K.G.), and by USDA-ARS Specific Cooperative Agreement 58-6615-4-237.

\section{REFERENCES}

Blomquist, G. J., Nelson, D. R., and De Renobales, M. 1987. Chemistry, biochemistry, and physiology of insect cuticular lipids. Arch. Insect Biochem. Physiol. 6:227-265.

BROWN, W. V., WATSON, J. A. L., and LACEY, M. J. 1994. The cuticular hydrocarbons of workers of three Australian Coptotermes species, C. michaelseni, C. brunneus and C. dreghorni (Isoptera: Rhinotermitidae). Sociobiology 23:277-291.

Brown, W. V., Watson, J. A. L., and LACEY, M. J. 1996. A chemosystematic survey using cuticular hydrocarbons of some species of the Australian harvester termite genus Drepanotermes (Isoptera: Termitidae). Sociobiology 27:199-221.

Chapman, R. F., Espelie, K. E., and Sword, G. A. 1995. Use of cuticular lipids in grasshopper taxonomy: A study of variation in Schistocerca shoshone (Thomas). Biochem. Syst. Ecol. 23:383-398.

CHILD, H. J. 1934. The internal anatomy of termites and the histology of the digestive tract, pp. 58-88, in C. A. Kofoid, S. F. Light, A. C. Horner, M. Randall, W. B. Herms, and E. E. Bowe (eds.). Termites and Termite Control, 2nd edn. University of California Press, Berkeley, CA. 
Clément, J.-L., Bagnères, A.-G., Uva, P., Wilfert, L., Quintana, A., Reinhard, J., and DronNET, S. 2001. Biosystematics of Reticulitermes termites in Europe: Morphological, chemical and molecular data. Insectes Soc. 48:202-215.

Collins, M. S., HAVERTY, M. I., and ThORNE, B. L. 1997. The termites (Isoptera: Kalotermitidae, Rhinotermitidae, Termitidae) of the British Virgin Islands: Distribution, moisture relations, and cuticular hydrocarbons. Sociobiology 30:63-76.

Coyne, J. A., Crittenden, A. P., and MaH, K. 1994. Genetics of a pheromonal difference contributing to reproductive isolation in Drosophila. Science 265:1461-1464.

EBELING, W. 1975. Urban Entomology. University of California Press, Los Angeles.

Espelie, K. E., Chapman, R. F., and Sword, G. A. 1994. Variation in the surface lipids of the grasshopper, Schistocerca americana (Drury). Biochem. Syst. Ecol. 22:563-575.

Grace, J. K., WoOdRow, R. J., and YATES, J. R. 2002. Distribution and management of termites in Hawaii. Sociobiology 40:87-93.

HADLEY, N. F. 1977. Epicuticular lipids of the desert tenebrionid beetle, Eleodes armata: Seasonal and acclimatory effects on composition. Insect Biochem. 7:277-283.

HADLEY, N. F. 1981. Cuticular lipids of terrestrial plants and arthropods: A comparison of their structure, composition, and waterproofing function. Biol. Rev. 56:23-47.

Hadley, N. F. 1985. The Adaptive Role of Lipids in Biological Systems. John Wiley and Sons, New York.

HAVERTY, M. I. and Nelson, L. J. 1997. Cuticular hydrocarbons of Reticulitermes (Isoptera: Rhinotermitidae) from northern California indicate undescribed species. Comp. Biochem. Physiol. 118B:869-880.

HAVERTY, M. I. and THORNE, B. L. 1989. Agonistic behavior correlated with hydrocarbon phenotypes in dampwood termites, Zootermopsis (Isoptera: Termopsidae). J. Insect Behav. 2:523543.

Haverty, M. I., Page, M., Nelson, L. J., and Blomquist, G. J. 1988. Cuticular hydrocarbons of dampwood termites, Zootermopsis: Intra- and intercolony variation and potential as taxonomic characters. J. Chem. Ecol. 14:1035-1058.

HAVERTY, M. I., ForsChlER, B. T., and Nelson, L. J. 1996a. An assessment of the taxonomy of Reticulitermes (Isoptera: Rhinotermitidae) from the southeastern United States based on cuticular hydrocarbons. Sociobiology 28:287-318.

HAVERTY, M. I., THORNE, B. L., and NeLSON, L. J. 1996b. Hydrocarbons of Nasutitermes acajutlae and comparison of methodologies for sampling cuticular hydrocarbons of Caribbean termites for taxonomic and ecological studies. J. Chem. Ecol. 22:2081-2109.

Haverty, M. I., Collins, M. S., Nelson, L. J., and Thorne, B. L. 1997. Cuticular hydrocarbons of termites of the British Virgin Islands. J. Chem. Ecol. 23:927-964.

HAVERTY, M. I., NeLSON, L. J., and ForsChLER, B. T. 1999. New cuticular hydrocarbon phenotypes of Reticulitermes (Isoptera: Rhinotermitidae) from the United States. Sociobiology 34:1-21.

Haverty, M. I., WoOdrow, R. J., Nelson, L. J., and Grace, J. K. 2000. Cuticular hydrocarbons of termites of the Hawaiian Islands. J. Chem. Ecol. 26:1167-1191.

HOWARD, R. W. 1998. Ontogenetic, reproductive, and nutritional effects on the cuticular hydrocarbons of the host-specific ectoparasitoid Cephalonomia tarsalis (Hymenoptera: Bethylidae). Ann. Entomol. Soc. Am. 91:101-112.

Howard, R. W., Thorne, B. L., Levings, S. C., and MCDaniel, C. A. 1988. Cuticular hydrocarbons as chemotaxonomic characters for Nasutitermes corniger (Motchulsky) and N. ephratae (Holmgren) (Isoptera: Termitidae). Ann. Entomol. Soc. Am. 81:395-399.

KaIB, M., BRANDL, R., and BAGINE, R. K. N. 1991. Cuticular hydrocarbon profiles: A valuable tool in termite taxonomy. Naturwissenschaften 78:176-179.

Moore, B. P. 1969. Biochemical studies in termites, pp. 407-432, in K. Krishna and F. M. Weesner (eds.). Biology of Termites, Vol. I. Academic Press, New York. 
Moore, H. B. 1992. An Introduction to Wood Destroying Insects: Their Identification, Biology, Prevention, and Control. Edgell Communications, Inc., Cleveland, OH.

Nelson, L. J., Cool, L. G., Forschler, B. T., and Haverty, M. I. 2001. Correspondence of soldier defense secretion mixtures with cuticular hydrocarbon phenotypes for chemotaxonomy of the termite genus Reticulitermes in North America. J. Chem. Ecol. 27:1449-1479.

NoIrot, C. 1969. Glands and secretion, pp. 89-123, in K. Krishna and F. M. Weesner (eds.). Biology of Termites, Vol. I. Academic Press, New York.

Noirot, C. and Noirot-TimothéE, C. 1969. The digestive system, pp. 49-88, in K. Krishna and F. M. Weesner (eds.). Biology of Termites, Vol. I. Academic Press, New York.

NutTING, W. L. 1966. Distribution and biology of the primitive dry-wood termite Pterotermes occidentis (Walker)(Kalotermitidae). Psyche 73:165-179.

Page, M., Nelson, L. J., Haverty, M. I., and BlomQuist, G. J. 1990a. Cuticular hydrocarbons of eight species of North American cone beetles, Conophthorus Hopkins. J. Chem. Ecol. 16:1173-1198.

Page, M., Nelson, L. J., Haverty, M. I., and BlomQuist, G. J. 1990b. Cuticular hydrocarbons as chemotaxonomic characters for bark beetles: Dendroctonus ponderosae, D. jeffreyi, D. brevicomis, and D. frontalis (Coleoptera: Scolytidae). Ann. Entomol. Soc. Am. 83:892-901.

Page, M., Nelson, L. J., Forschler, B. T., and Haverty, M. I. 2002. Cuticular hydrocarbons suggest three lineages in Reticulitermes (Isoptera: Rhinotermitidae) from North America. Comp. Biochem. Physiol. 131B:305-324.

Page, R. E., Metcalf, R. A., Metcalf, R. L., Erickson, E. H. JR., and lampman, R. L. 1991. Extractable hydrocarbons and kin recognition in honeybee (Apis mellifera L.). J. Chem. Ecol. 17:745-756.

Pomonis, J. G., Fatland, C. F., Nelson, D. R., and ZaYlskie, R. G. 1978. Insect hydrocarbons. Corroboration of structure by synthesis and mass spectrometry of mono- and dimethylalkanes. J. Chem. Ecol. 4:27-38.

R Development Core Team, 2004. A language and environment for statistical computing. R Foundation for Statistical Computing, Vienna, Austria. ISBN 3-900051-07-0, URL http:// www.R-project.org.

Romoser, W. S. and Stoffolano, J. G. JR. 1988. The Science of Entomology, 4th edn. McGrawHill, Boston, MA.

Schal, C., Sevala, V. L., Young, H., and Bachmann, J. A. S. 1998. Synthesis and transport of hydrocarbons: Cuticle and ovary as target tissues. Am. Zool. 38:382-393.

Scheffrahn, R. H., Darlington, J. P. E. C., Collins, M. S., KreceK, J., and Su, N.-Y. 1994. Termites (Isoptera: Kalotermitidae, Rhinotermitidae, Termitidae) of the West Indies. Sociobiology 24:213-238.

Scheffrahn, R. H., Su, N.-Y., Chase, J. A., Mangold, J. R., Grace, J. K., and Yates, J. R. III. 2000. First record of Cryptotermes cynocephalus Light (Isoptera: Kalotermitidae) and natural woodland infestations of C. brevis (Walker) on Oahu, Hawaiian Islands. Proc. Hawaii. Entomol. Soc. 34:141-146.

Sevala, V. L., Bagneres, A.-G., Kuenzli, M., BlomQuist, G. J., and Schal, C. 2000. Cuticular hydrocarbons of the dampwood termite, Zootermopsis nevadensis: Caste differences and role of lipophorin in transport of hydrocarbons and hydrocarbon metabolites. J. Chem. Ecol. 26:765-789.

SNODGRASS, R. E. 1935. Principles of Insect Morphology. McGraw-Hill, New York.

Souto, L. and KitAYAMA, K. 2000. Constrictotermtes cyphergaster (Isoptera: Termitidae: Nasutitermitinae) maintain foraging trails for a longer period by means of fecal droplets. Sociobiology 35:367-372.

TAKEMATSU, Y. and YAMAOKA, R. 1999. Cuticular hydrocarbons of Reticulitermes (Isoptera: 
Rhinotermitidae) in Japan and neighboring countries as chemotaxonomic characters. Appl. Entomol. Zool. 3:179-188.

THORNE, B. L. and HAVERTY, M. I. 1989. Accurate identification of Zootermopsis species (Isoptera: Termopsidae) based on a mandibular character of nonsoldier castes. Ann. Entomol. Soc. Am. $82: 262-266$.

Thorne, B. L., Haverty, M. I., Page, M., and Nutting, W. L. 1993. Distribution and biogeography of the North American termite genus Zootermopsis (Isoptera: Termopsidae). Ann. Entomol. Soc. Am. 86:532-544.

TOOLSON, E. C. and KUPER-SIMBRÓN, R. 1989. Laboratory evolution of epicuticular hydrocarbon composition and cuticular permeability in Drosophila pseudoobscura: Effects on sexual dimorphism and thermal-acclimation ability. Evolution 43:468-473.

Watson, J. A. L., Brown, W. V., Miller, L. R., Carter, F. L., and Lacey, M. J. 1989. Taxonomy of Heterotermes (Isoptera: Rhinotermitidae) in southeastern Australia: Cuticular hydrocarbons of workers, and soldier and alate morphology. Syst. Entomol. 14:299-325.

WeEsNer, F. M. 1970. Termites of the Nearctic Region, pp. 477-525, in K. Krishna and F. M. Weesner (eds.). Biology of Termites, Vol. II. Academic Press, New York.

Woodrow, R. J., Grace, J. K., Nelson, L. J., and HAVERTY, M. I. 2000. Modification of cuticular hydrocarbons of Cryptotermes brevis (Isoptera: Kalotermitidae) in response to temperature and relative humidity. Environ. Entomol. 29:1100-1107.

Zimmerman, E. C. 1948. Insects of Hawaii, Vol. 2. University of Hawaii Press, Honolulu, HI. 